\title{
Towards a Standardized Procedure for the Production of Infective Spores to Study the Pathogenesis of Dermatophytosis
}

\author{
Emilie Faway ${ }^{1, \dagger}$, Cindy Staerck ${ }^{2,+}$, Célya Danzelle ${ }^{2}$, Sophie Vroomen ${ }^{2}$, Christel Courtain ${ }^{1}$, Bernard Mignon ${ }^{2, *, \ddagger}$ \\ and Yves Poumay $1, *, \ddagger(D)$ \\ 1 Molecular Physiology Research Unit, NAmur Research Institute for Life Sciences (URPHYM-NARILIS), \\ Faculty of Medicine, University of Namur, 5000 Namur, Belgium; emilie.faway@unamur.be (E.F.); \\ christel.courtain@student.unamur.be (C.C.) \\ 2 Fundamental and Applied Research for Animals \& Health (FARAH), Faculty of Veterinary Medicine, \\ University of Liège, 4000 Liège, Belgium; cindy.staerck@uliege.be (C.S.); celya.danzelle@uliege.be (C.D.); \\ sophie.vroomen@uliege.be (S.V.) \\ * Correspondence: bmignon@uliege.be (B.M.); yves.poumay@unamur.be (Y.P.); Tel.: +32-4-366-40-99 (B.M.); \\ +32-81-72-42-57 (Y.P.) \\ + These authors contributed equally to this work. \\ $\ddagger$ These authors contributed equally to this work.
}

check for

updates

Citation: Faway, E.; Staerck, C.; Danzelle, C.; Vroomen, S.; Courtain,

C.; Mignon, B.; Poumay, Y. Towards a Standardized Procedure for the Production of Infective Spores to Study the Pathogenesis of Dermatophytosis. J. Fungi 2021, 7, 1029. https://doi.org/10.3390/ jof7121029

Academic Editor:

Anuradha Chowdhary

Received: 28 October 2021

Accepted: 26 November 2021

Published: 30 November 2021

Publisher's Note: MDPI stays neutral with regard to jurisdictional claims in published maps and institutional affiliations.

Copyright: (C) 2021 by the authors Licensee MDPI, Basel, Switzerland. This article is an open access article distributed under the terms and conditions of the Creative Commons Attribution (CC BY) license (https:// creativecommons.org/licenses/by/ $4.0 /)$.
Abstract: Dermatophytoses are superficial infections of human and animal keratinized tissues caused by filamentous fungi named dermatophytes. Because of a high and increasing incidence, as well as the emergence of antifungal resistance, a better understanding of mechanisms involved in adhesion and invasion by dermatophytes is required for the further development of new therapeutic strategies. In the last years, several in vitro and in vivo models have emerged to study dermatophytosis pathogenesis. However, the procedures used for the growth of fungi are quite different, leading to a highly variable composition of inoculum for these models (microconidia, arthroconidia, hyphae), thus rendering difficult the global interpretation of observations. We hereby optimized growth conditions, including medium, temperature, atmosphere, and duration of culture, to improve the sporulation and viability and to favour the production of arthroconidia of several dermatophyte species, including Trichophyton rubrum and Trichophyton benhamiae. The resulting suspensions were then used as inoculum to infect reconstructed human epidermis in order to validate their ability to adhere to and to invade host tissues. By this way, this paper provides recommendations for dermatophytes culture and paves the way towards a standardized procedure for the production of infective spores usable in in vitro and in vivo experimental models.

Keywords: dermatophytosis; dermatophytes; infective spores; arthroconidia; microconidia; reconstructed human epidermis; dermatophyte pathogenesis; experimental models; Trichophyton; Microsporum

\section{Introduction}

Dermatophytoses are superficial infections of the skin, hair, and nails due to filamentous and keratinolytic fungi named dermatophytes [1]. Currently, dermatophytoses constitute the most common mycosis worldwide, affecting 20-25\% of the global population [2], and up to 50\% among populations at risk, such as sportsmen and diabetic patients [3-5]. Genetic predispositions also exist and heredity participates, along with lifestyle, in the increase of dermatophytosis prevalence observed in the last decades [6,7]. Classical antifungals, such as azole derivatives and terbinafine, are generally efficient to treat dermatophytosis [8], but their use is associated with potential toxicity when systemic administration is required [9] and must cope with the growing emergence of resistant strains [10-14].

Because of this high and still increasing prevalence of dermatophytosis, as well as the difficulties encountered with currently available treatments, more research is needed to 
elucidate mechanisms responsible for fungal adherence and invasion of the host tissue. For this purpose, several in vitro and in vivo models of infection by dermatophytes have been developed (for reviews, see $[15,16]$ respectively).

Experimental infection models described in the literature use different compositions of inoculum, consisting in suspensions enriched in unicellular infective spores, namely microconidia [17-23] or arthroconidia [24-29], or in a mix of microconidia and hyphae [30-35]. Microconidia, as well as macroconidia, are a type of thallic spores resulting from lateral or terminal budding of hyphae. Although micro- and macroconidia are easier to produce in culture, and thus frequently used as infective spores in most experimental models, they have never been observed in natural lesions [36]. In contrast, arthroconidia, produced by fragmentation and disarticulation of hyphae septate $[37,38]$, are recognized as natural vectors of dermatophytosis [36]. However, even though their use appears more representative of natural infection, arthroconidia are less frequently used in experimental models since their formation is particularly difficult to induce in vitro. Arthroconidia production was nevertheless documented in some studies using T. mentagrophytes $[37,39-42]$ or M. canis [25], and more recently with T. rubrum [43,44].

This heterogeneity in the composition of inoculum, produced in various conditions of culture, including media, temperature, atmosphere, or duration, could influence the development of infection, rendering impossible any comparison between the observations obtained from different experimental models. Thus, overall conclusions about the pathogenic mechanisms responsible for dermatophytosis remain hard to define. In this paper, we assessed the growth and production of spores, especially arthroconidia, by several dermatophyte species frequently isolated from human lesions, in different culture conditions, with the aim to define a standardized procedure for the production of infective spores usable as inoculum in experimental in vitro and in vivo models. In addition, the infectious potential of these inocula was checked through an evaluation of infection development in a model of reconstructed human epidermis (RHE).

\section{Materials and Methods}

\subsection{Dermatophyte Strains}

This study was conducted on a panel of 13 dermatophyte strains described in Table 1 and obtained from IHEM (Institute of Hygiene and Epidemiology-Mycology section) from the Scientific Institute of Public Health (Brussels, Belgium). The strains were firstly grown on a homemade Sabouraud (SAB) medium (Table 2) plate at $30^{\circ} \mathrm{C}$ for 14 days.

\subsection{Growth Evaluation}

The growth capacity of dermatophytes was determined using five different agar media: Sabouraud (SAB), potato dextrose agar (PDA), yeast peptone dextrose (YPD), malt medium (MALT), and yeast extract nitrogen (YEN). The composition of each medium, as well as the suppliers for all ingredients used, are described in Table 2. Practically, fungi were grown on $\mathrm{SAB}$ at $30^{\circ} \mathrm{C}$ for 14 days, after which the fungal material was scraped with a scalpel blade and transferred in PBS ( $\mathrm{pH}$ 7.2). Fungal suspensions were centrifuged at $3000 \times g$ for $5 \mathrm{~min}$, before pellets were washed three times with PBS and finally resuspended in an adequate volume of PBS to obtain a concentration of $1 \times 10^{5}$ colony-forming unit (CFU) per $\mathrm{mL}$. Five microliters of the resulting fungal suspension were then spotted on the centre of each medium plate and incubated in the dark at $30^{\circ} \mathrm{C}$. The diameter of fungal colonies was measured every day for 31 days and radial growths were determined during the exponential phase by analysis of the growth curves. 
Table 1. Dermatophyte isolates analysed in this study. Information on each isolate was retrieved from the online catalogue of Belgian Coordinated Collections of Micro-organisms (BCCM)/Institute of Hygiene and Epidemiology-Mycology section (IHEM; https:/ / bccm.belspo.be/catalogues/ihem-catalogue-search; accessed on 1 September 2021).

\begin{tabular}{|c|c|c|c|c|}
\hline \multirow{2}{*}{ Genus-Species-Isolate Number } & \multicolumn{4}{|c|}{ Isolation } \\
\hline & Year & Lesion & Host & Country \\
\hline \multicolumn{5}{|l|}{ Microsporum } \\
\hline M. audouinii IHEM 10316 & 1997 & Tinea capitis & Human & Belgium \\
\hline M. canis IHEM 22957 & 2009 & Tinea & Cat & Belgium \\
\hline M. canis IHEM 22958 & 2009 & Tinea & Cat & Belgium \\
\hline M. canis IHEM 21239 & 2005 & Tinea & Cat & Belgium \\
\hline \multicolumn{5}{|l|}{ Trichophyton } \\
\hline T. benhamiae IHEM 20163 & 2002 & Tinea corporis & Human & Switzerland \\
\hline T. benhamiae IHEM 20161 & 2002 & Tinea faciei & Human & Switzerland \\
\hline T. interdigitale IHEM 00584 & 1981 & Tinea pedis & Human & Belgium \\
\hline T. mentagrophytes IHEM 22740 & 2008 & Skin & Human & Switzerland \\
\hline T. mentagrophytes IHEM 22733 & 2008 & Tinea & Cat & Switzerland \\
\hline T. mentagrophytes IHEM 25841 & 2012 & Skin and hairs & Dog & Belgium \\
\hline \multicolumn{5}{|l|}{ T. mentagrophytes TIMM $2789^{1}$} \\
\hline T. rubrum IHEM 13894 & 1985 & Tinea cruris and onychomycosis & Human & Democratic Republic of the Congo \\
\hline T. tonsurans IHEM 24958 & 2008 & & Human & Belgium \\
\hline
\end{tabular}

${ }^{1}$ The strain T. mentagrophytes TIMM 2789 was gracefully supplied by T. Yamada from the Institute of Medical Mycology, Teikyo University [45].

Table 2. Composition of agar media used in this study.

\begin{tabular}{cccccc}
\hline Composition in g/L & $\begin{array}{c}\text { Sabouraud } \\
\text { (SAB) }\end{array}$ & $\begin{array}{c}\text { Potato Dextrose } \\
\text { Agar (PDA) }\end{array}$ & $\begin{array}{c}\text { Yeast Peptone } \\
\text { Dextrose (YPD) }\end{array}$ & $\begin{array}{c}\text { Malt Medium } \\
\text { (MALT) }\end{array}$ & $\begin{array}{c}\text { Yeast Extract } \\
\text { Nitrogen (YEN) }\end{array}$ \\
\hline Tryptone $^{1}$ & 10 & & 20 & 6 \\
\hline Glucose $^{2}$ & 20 & & & \\
\hline Potato dextrose agar $^{3}$ & & 39 & 20 & \\
\hline Malt extract $^{4}$ & & & & \\
\hline Yeastextract $^{5}$ & 20 & 5 & 20 & 20 \\
\hline Agar & & & & \\
\hline pH & $7.5 \pm 0.3$ & $5.4 \pm 0.5$ & $7.5 \pm 0.1$ & $6.1 \pm 0.2$ \\
\hline
\end{tabular}

${ }^{1}$ Tryptone (peptone from casein) cat. no. 84610.0500; VWR Chemicals, Radnor, PA, USA; ${ }^{2} \mathrm{D}(+)$-Glucose monohydrate, + de $99 \%$, extra pur cat. no. 450740010; Acros-organics B.V.B.A, Fisher Scientific, Fair Lawn, NJ, USA; ${ }^{3}$ Potato dextrose agar cat. no. 110130; Merck KGaA, Darmstadt, Germany; ${ }^{4}$ Malt Extract cat. no. 1.05391.0500; Merck KGaA, Darmstadt, Germany; ${ }^{5}$ Yeast extract cat. no. 84601.0500; VWR Chemicals, Radnor, PA, USA; ${ }^{6}$ Agar cat. no. BP-1423-2; VWR Chemicals, Radnor, PA, USA.

\subsection{Sporulation Evaluation}

The sporulation of dermatophytes was assessed after 31 days of growth at $30{ }^{\circ} \mathrm{C}$ on the five used agar media (SAB, PDA, YPD, MALT and YEN, Table 2). Practically, the fungal material was scrapped from the surface of the agar plate with a scalpel blade and suspended in PBS ( $\mathrm{pH}$ 7.2). Recovered suspensions were then filtered through three Miracloth layers (22-25 $\mu \mathrm{m}$ pore size; cat. no. 475855 ; Merck KGaA, Darmstadt, Germany) to remove hyphae and centrifuged at $3000 \times g$ for $5 \mathrm{~min}$. Pellets were washed three times with PBS and resuspended in $5 \mathrm{~mL}$ PBS. Total spore concentration, including microconidia, macroconidia, and arthroconidia, was microscopically enumerated using a Thoma cell counting chamber. 


\subsection{Production of Infective Spore Suspensions}

The production of a suspension of infective spores adequate for use in experimental models was evaluated on three different homemade media (SAB, PDA and YEN, Table 2). Briefly, after growth on $\mathrm{SAB}$ for 14 days, the fungal material was scraped with a scalpel blade, transferred in PBS ( $\mathrm{pH} 7.2$ ), and seeded on SAB, PDA, or YEN media for sporulation. The plates were incubated at $30{ }^{\circ} \mathrm{C}$ under $12 \% \mathrm{CO}_{2}$ or at $37^{\circ} \mathrm{C}$ under $10 \% \mathrm{CO}_{2}$, for 10,21 , or 31 days. Spore suspensions were then recovered as previously described in Section 2.3, with an additional step of stirring in order to efficiently dissociate arthroconidia and microconidia from hyphae.

The viable fungal concentration of the resulting suspensions was determined by CFU counting of serial dilutions after three days of incubation at $30{ }^{\circ} \mathrm{C}$ on $\mathrm{SAB}$, and the total spore concentration was determined under light microscopy as described in Section 2.3. Simultaneously, the proportion of arthroconidia was calculated relative to the total spore concentration after examining five randomly selected microscopical fields under $\times 400$ magnification. The spore suspensions were finally observed and pictured under differential interference contrast (DIC) microscopy (Olympus; Tokyo, Japan).

\subsection{Infection Development on Reconstructed Human Epidermis}

Initially, the inoculum used in our model of infection on RHE consisted in a suspension of spores recovered after a 21-days incubation on YEN at $30^{\circ} \mathrm{C}$ under $12 \% \mathrm{CO}_{2}$ [28]. Based on our previous results, herein, we compared inoculum prepared using this conventional procedure with a spore suspension recovered after a 10-days incubation on PDA at $30^{\circ} \mathrm{C}$ under $12 \% \mathrm{CO}_{2}$, appearing as the optimal method to obtain a high viable fungal concentration. The preparation of these spore suspensions was performed as described in Section 2.4 Spores were stored at $4{ }^{\circ} \mathrm{C}$ and used within one month.

The assays of infection on RHE were performed for T. benhamiae IHEM 20163 and T. rubrum IHEM 13894 strains. The inoculum was adjusted at the required quantity by dilution in PBS: 30 CFU for T. benhamiae and $1000 \mathrm{CFU}$ for T. rubrum, respectively corresponding to densities of about 53 per $\mathrm{cm}^{2}$ and 1700 per $\mathrm{cm}^{2}$.

\subsubsection{Production of RHE and Infection by Dermatophytes}

RHE were prepared as previously described [46]. Briefly, normal human keratinocytes were isolated from skin samples obtained from plastic surgery (Dr. Bienfait and Dr. Blairvacq, Clinique St-Luc, Namur-Bouge, Belgium). After prior amplification in EpiLife medium (cat. no. M-EPI-500-CA; Life Technologies, Waltham, MA, USA) supplemented with Human Keratinocyte Growth Supplement (HKGS; cat. no. S-001-5; Life Technologies, Waltham, MA, USA), primary keratinocytes were seeded at a high density of 250,000 cells per $\mathrm{cm}^{2}$ onto polycarbonate culture insert characterized by pores of $0.4 \mu \mathrm{m}$ diameter (cat. no. PIHP01250; Merck KGaA, Darmstadt, Germany) in EpiLife medium supplemented with HKGS and $1.5 \mathrm{mM} \mathrm{Ca}^{2+}$. After $24 \mathrm{~h}$, keratinocytes were exposed to the air-liquid interface by removing the culture medium above the insert, while the medium under the insert was replaced by EpiLife medium supplemented with HKGS, $1.5 \mathrm{mM} \mathrm{Ca}^{2+}$, $10 \mathrm{ng} / \mu \mathrm{L}$ keratinocytes growth factor (KGF; cat. no. 251-KG; R\&D Systems, Abingdon, UK) and $50 \mu \mathrm{g} / \mathrm{mL}$ vitamin C (2-phospho-L-ascorbic acid trisodium salt; cat. no. 49752; Merck KGaA, Darmstadt, Germany). Keratinocytes were maintained at $37{ }^{\circ} \mathrm{C}$ in a humidified atmosphere containing $5 \% \mathrm{CO}_{2}$ with medium under the insert renewed every two days. After 11 days, fully differentiated epidermis can be obtained and used for infection by dermatophytes.

Infection of RHE by dermatophytes was performed as described in Faway et al. [28]. Practically, an inoculum made of infective spore suspensions, produced as depicted in Section 2.4, was topically applied onto the surface of RHE on the day of infection. At this step, an additional inoculum was seeded onto $\mathrm{SAB}$ plates to monitor the fungal load by counting $\mathrm{CFU}$ after three days of incubation at $30^{\circ} \mathrm{C}$ (internal control). Four hours after infection, the surface of RHE was rinsed three times with PBS in order to eliminate non- 
adherent spores and to expose again the cultured keratinocytes to the air-liquid interface. Non-adherent spores recovered during washes were counted onto SAB, in order to evaluate the fraction of remaining adherent spores. Finally, infected RHE were maintained in culture for four additional days with culture medium and the tissue was renewed every day.

\subsubsection{Histological Analysis}

Infected RHE were recovered each day during the four days following infection, fixed in $4 \%$ formaldehyde solution, and histologically processed before periodic-acid Schiff (PAS) staining with pre-treatment using $\alpha$-amylase and hemalun counterstaining, as described by Faway et al. [28].

\subsubsection{Measurement of Infection Level}

The quantification of the infection level on RHE was performed by the assessment of the copy number of the dermatophyte $18 \mathrm{~S}$ rDNA gene using qPCR as previously described [28]. Briefly, total DNA was extracted from infected RHE using the DNeasy ${ }^{\circledR}$ Blood \& Tissue Kit (cat. no. 69504; Qiagen, Hilden, Germany) after tissue homogenization using Tissue Grinder (cat. no. NG010; NIPPON Genetics EUROPE, Duren, Germany). Quantitative PCR was then performed using Light Cycler 96 (Roche, Basel, Switzerland) and the PCR mixture was made of Takyon ${ }^{\mathrm{TM}}$ No ROX SYBR ${ }^{\circledR}$ Master Mix (cat. no. UF-NSMT-B0701; Eurogentec, Seraing, Belgium), $300 \mathrm{nM}$ of each primer, and $20 \mathrm{ng}$ of DNA. Primer sequences were chosen to amplify the $18 \mathrm{~S}$ rDNA gene from several dermatophyte species, including T. rubrum and T. benhamiae (18SrDNA-F 5'-TAACGAACGAGACCTTAACC- $3^{\prime}$ and 18SrDNA-R 5'-TTATTGCCTCAAACTTCCAT- $3^{\prime}$, described in Paugam et al. [47]). Absolute quantification was realized thanks to a standard curve made of known 18S rDNA copy number samples. These samples were obtained after DNA extraction from T. rubrum or T. benhamiae mycelium grown on SAB plates, PCR amplification of the $18 \mathrm{~S}$ rDNA gene using the Platinum ${ }^{\mathrm{TM}}$ SuperFi ${ }^{\mathrm{TM}}$ DNA Polymerase (cat. no. MAN0014882; Invitrogen, Carlsbad, CA, USA), and purification by the MinElute ${ }^{\circledR}$ PCR Purification Kit (cat. no. 28004; Qiagen, Hilden, Germany). For more details, please refer to Faway et al. [28].

\subsubsection{Epidermal Barrier Integrity Monitoring}

The integrity of the RHE epidermal barrier was monitored during infection by measurement of the trans-epithelial electrical resistance (TEER) using a Millicell ERS-2 Voltohmmeter (cat. no. MERS00002; Merck KGaA, Darmstadt, Germany). These measurements are expressed as a percent of TEER values measured through RHE before infection.

\subsubsection{RNA Extraction, Reverse Transcription and Quantitative PCR}

Expression of proinflammatory cytokines and antimicrobial peptides by keratinocytes during infection of RHE was assessed by RT-qPCR after total RNA extraction. RPLP0 was used as a reference gene [48] and results are expressed as relative expression compared to control non-infected RHE. Practically, total RNA was extracted from infected and control RHE using RNeasy Mini Kit (cat. no. 74104; Qiagen, Hilden, Germany) according to the manufacturer's instructions, and $1 \mu \mathrm{g}$ of RNA was reverse-transcribed into cDNA using the SuperScript III Reverse Transcriptase kit (cat. no. 18080; Invitrogen, Carlsbad, CA, USA). The qPCR mixture contained Takyon ${ }^{\mathrm{TM}}$ No ROX SYBR ${ }^{\circledR}$ Master Mix (cat. no. UF-NSMT-B0701; Eurogentec, Seraing, Belgium), $300 \mathrm{nM}$ of each primer and $5 \mu \mathrm{L}$ of cDNA diluted 1:20 in distilled water. The qPCR was performed using Light $\mathrm{Cy}-$ cler 96 (Roche, Basel, Switzerland). All primers used for qPCR were designed in-house using Primer-BLAST online software (https:/ / www.ncbi.nlm.nih.gov/tools/primer-blast/ accessed on 1 September 2021) and showed efficacy between 90\% and 110\% [29]. Primers sequences are listed in Table 3. 
Table 3. Sequences of primer pairs used for RT-qPCR.

\begin{tabular}{|c|c|c|}
\hline Gene Symbol & Forward Primer & Reverse Primer \\
\hline BD2 & 5'-ATCAGCCATGAGGGTCTTGT-3' & 5'-GAGACCACAGGTGCCAATTT-3' \\
\hline BD3 & 5'-TCCAGGTCATGGAGGAATCAT-3' & 5'-CGAGCACTTGCCGATCTGT-3' \\
\hline IL- $1 \alpha$ & 5'-AACCAGTGCTGCTGAAGGAGAT-3' & 5'-TGGTCTCACTACCTGTGATGGTTT-3' \\
\hline IL-1 $\beta$ & 5'-TCCCCAGCCCTTTTGTTGA-3' & 5'-TTAGAACCAAATGTGGCCGTG-3' \\
\hline IL-8 & 5'-GCAGAGGGTTGTGGAGAAGTTT-3' & 5'-TTGGATACCACAGAGAATGAATTTTT-3' \\
\hline LL-37 & 5'-CCAGGACGACACAGCAGTCA-3' & 5'-CTTCACCAGCCCGTCCTTC-3' \\
\hline RPLP0 & 5'-ATCAACGGGTACAAACGAGTC-3' & 5'-CAGATGGATCAGCCAAGAAGG-3' \\
\hline $\mathrm{TNF} \alpha$ & 5'-GAGGCCAAGCCCTGGTATG-3' & 5'-CGGGCCGATTGATCTCAGC-3' \\
\hline
\end{tabular}

\subsubsection{ELISA Assays}

The release of the proinflammatory cytokines IL- $1 \alpha$ and IL- 8 by keratinocytes during infection of RHE was monitored by ELISA assay performed on culture medium. Commercial ELISA kits, Human IL-1 alpha/IL1F1 DuoSet ELISA and Human IL-8/CXCL8 DuoSet ELISA (cat. no. DY200 and DY208; R\&D Systems, Abingdon, UK), were used according to the manufacturer's instructions.

\subsection{Statistical Analysis}

Results for each assay are expressed as the means with standard deviations, or individual values, of at least triplicate experiments performed independently and using freshly prepared fungal suspensions. Data were plotted using GraphPad Prism 9 software. Results were analysed by one-way (ANOVA1) or two-way (ANOVA2) variance analysis using GraphPad Prism 9 and SigmaPlot version 11.0 software, and were considered as significantly different with a $p$-value $<0.05$.

\section{Results}

\subsection{Growth and Sporulation of Dermatophytes Are Improved on PDA}

The growth of dermatophytes was monitored on different media (SAB, PDA, YPD, MALT, and YEN) by determination of colony diameter and radial growth. Strangely, YEN medium, classically used for the production of spore suspensions of M. canis appeared not suitable for Trichophyton species (Figure S1B). Globally, the colony diameters of all the strains used in this study were higher on PDA than on the other media (Figure S1A) and these data were in accordance with the radial growth determined on PDA (Figure S2). Notably, T. rubrum IHEM 13894 presented a diameter about twofold superior on SAB and PDA as compared to other media, without significant improvement of the radial growth (Figures 1A,B and S3). For T. benhamiae IHEM 20163, the colony size after 10 days was even more enhanced on PDA than on SAB (Figures 2A,B and S3). In addition, all the strains, except M. audouinii IHEM 10316, T. rubrum IHEM 13894, and T. tonsurans IHEM 24958, exhibited a reproductive fungal growth curve on PDA, with few variations between replicate assays. 

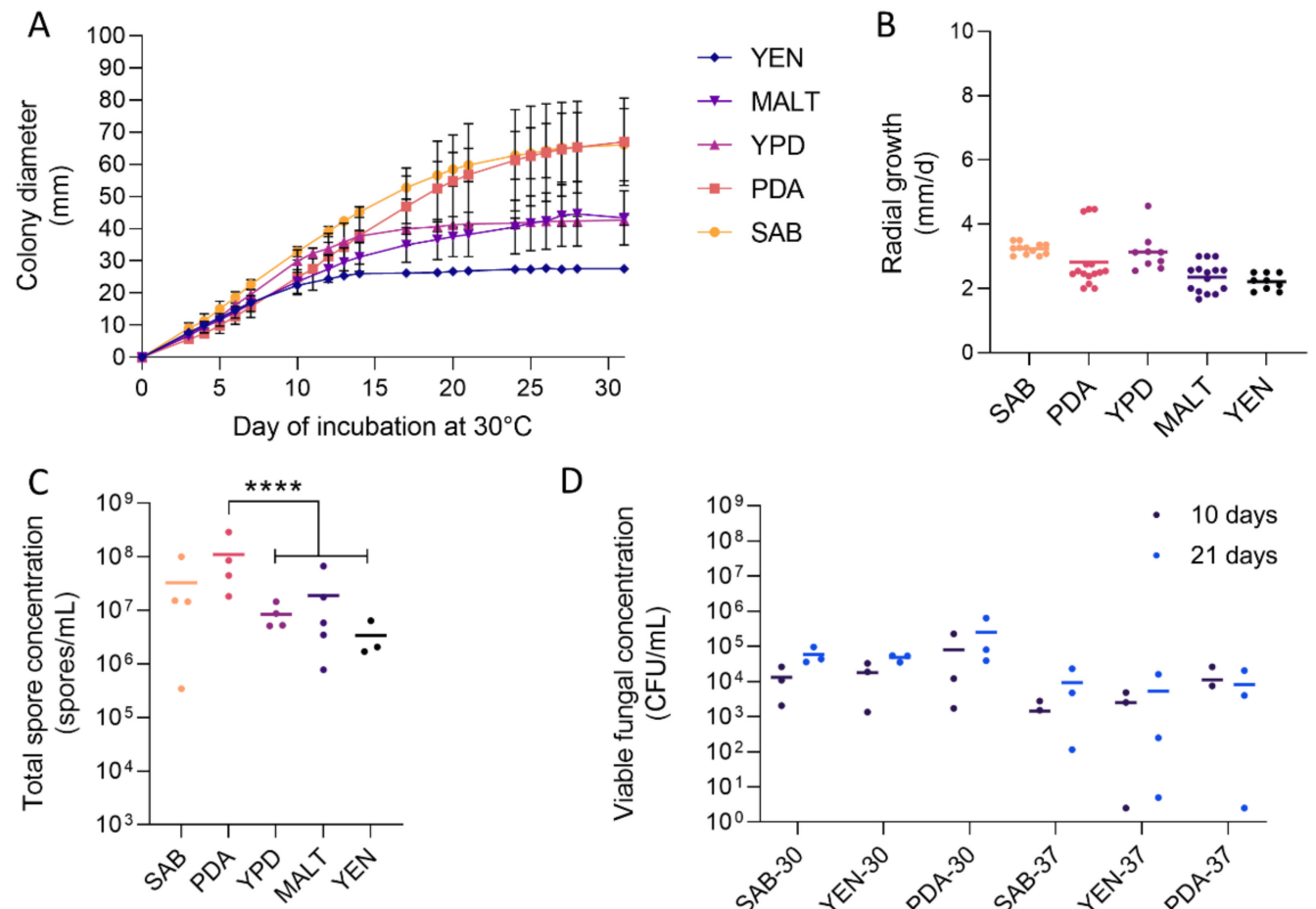

D

$$
\begin{aligned}
& \rightarrow \text { YEN } \\
& \rightarrow \text { MALT } \\
& \leftarrow \text { YPD } \\
& \rightarrow \text { PDA } \\
& \rightarrow \text { SAB }
\end{aligned}
$$

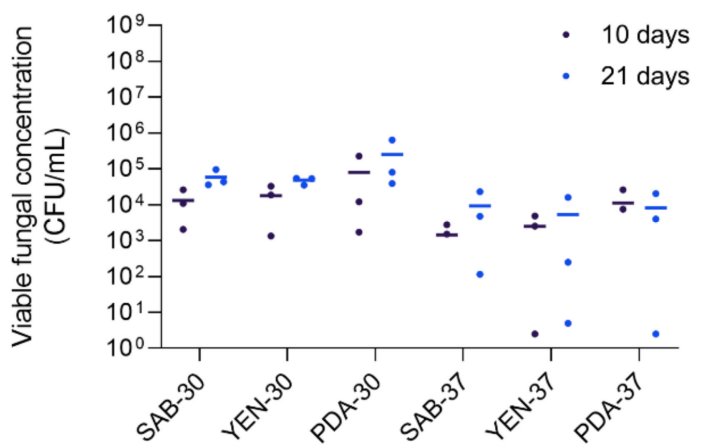

E

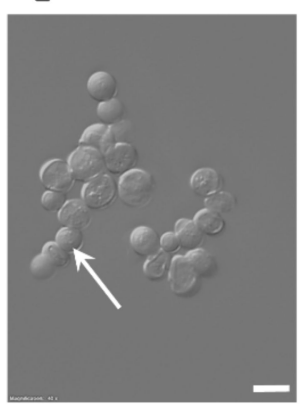

F

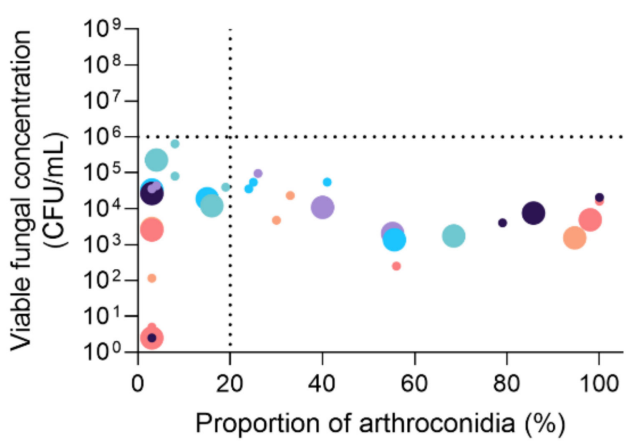

Conditions Time

SAB-30 (days)

YEN-30 -21

PDA-30 10

YEN-37

- SAB-37

- PDA-37

Figure 1. Growth and sporulation of T. rubrum IHEM 13894. T. rubrum IHEM 13894 were seeded over five agar media, Sabouraud (SAB), Potato Dextrose Agar (PDA), Yeast Peptone Dextrose (YPD), Malt medium (MALT) or Yeast Extract Nitrogen (YEN), and grown at $30^{\circ} \mathrm{C}$ for 31 days. Fungal material was then scrapped, suspended in PBS and filtered to obtain fungal suspensions. (A) Diameter of fungal colonies was measured daily, (B) radial growth was determined during the exponential phase of growth, and $(\mathbf{C})$ the total spore concentration recovered in fungal suspension was determined by counting using a Thoma chamber under light microscopy. To promote sporulation and arthroconidial production by T. rubrum IHEM 13894, fungi were seeded on SAB, PDA or YEN media, and incubated at $30{ }^{\circ} \mathrm{C}$ under $12 \% \mathrm{CO}_{2}$ or at $37{ }^{\circ} \mathrm{C}$ under $10 \% \mathrm{CO}_{2}$, for 10 or 21 days. Fungal material was then scrapped, suspended in PBS, stirred and filtered to obtain spore suspensions. (D) Viable fungal concentration was determined by counting colony-forming units (CFU). (E) Morphology of arthroconidia (white arrow) was observed using differential interference contrast (DIC) microscopy. Scale bar $=10 \mu \mathrm{m}$. (F) Viable fungal concentration was correlated with the proportion of arthroconidia relative to the total number of fungal elements, determined by counting using a Thoma chamber under light microscopy. Dotted lines indicate the minimal viable fungal concentration required for further use on experimental models, based on the most important published models $[15,16]$ and personal observations, as well as the proportion of $20 \%$ arthroconidia, arbitrarily chosen as a minimal value for suspensions enriched in arthroconidia. Data analysis: $n \geq 3$; means \pm SD for (A), means and individual values for (B-D), and single plots for (F); ANOVA2 for (B-D) compared to PDA; ${ }^{* * * *} p<0.0001$. 


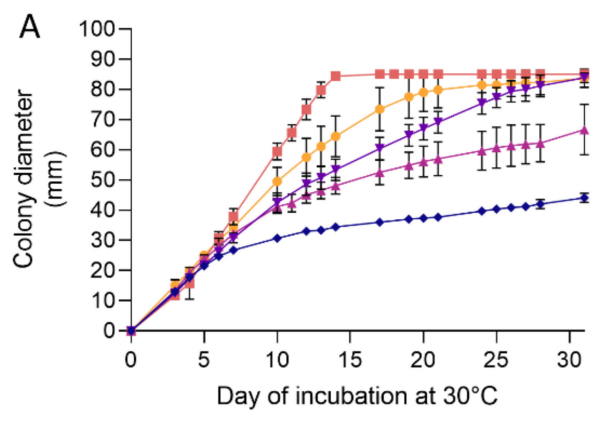

B
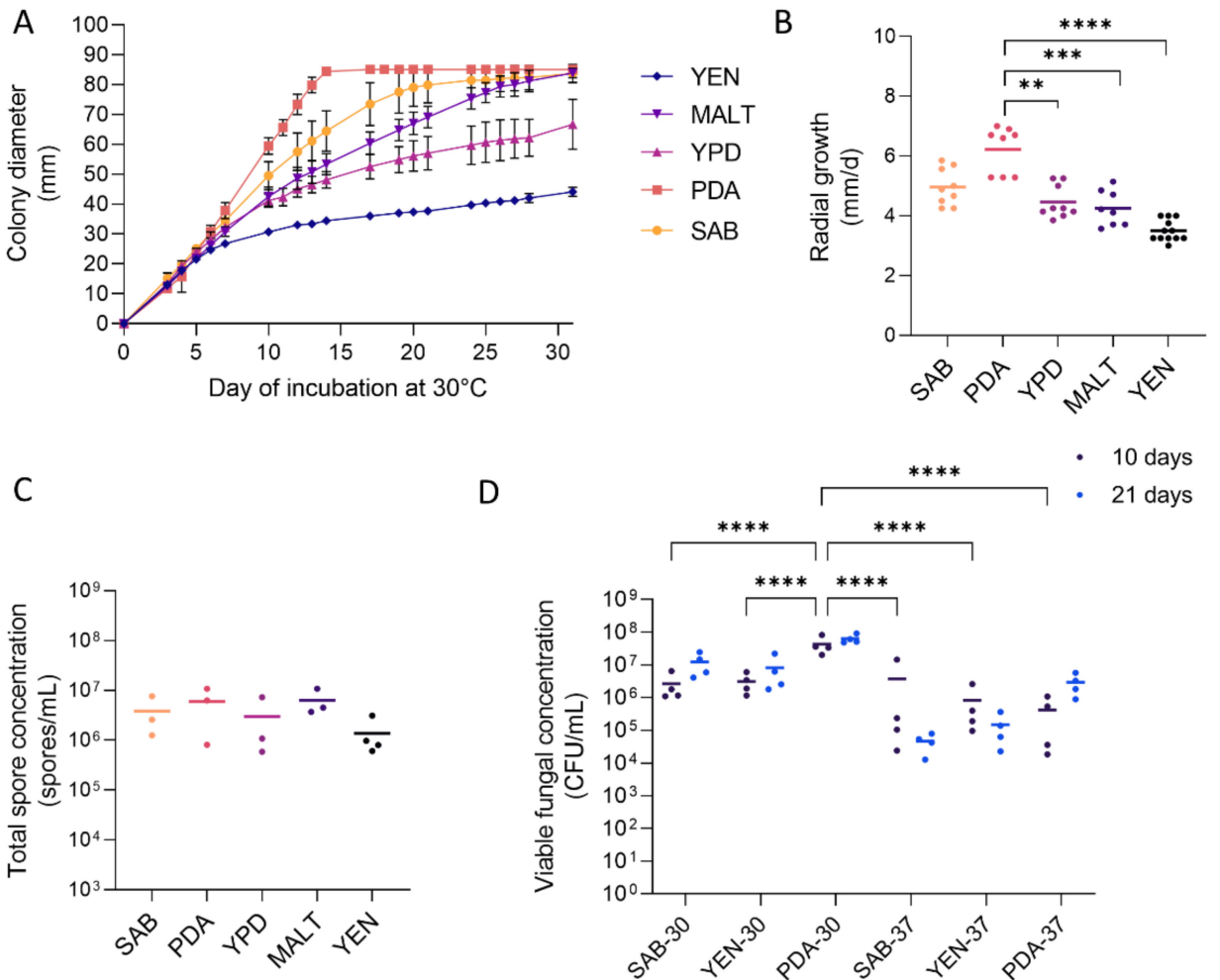

D
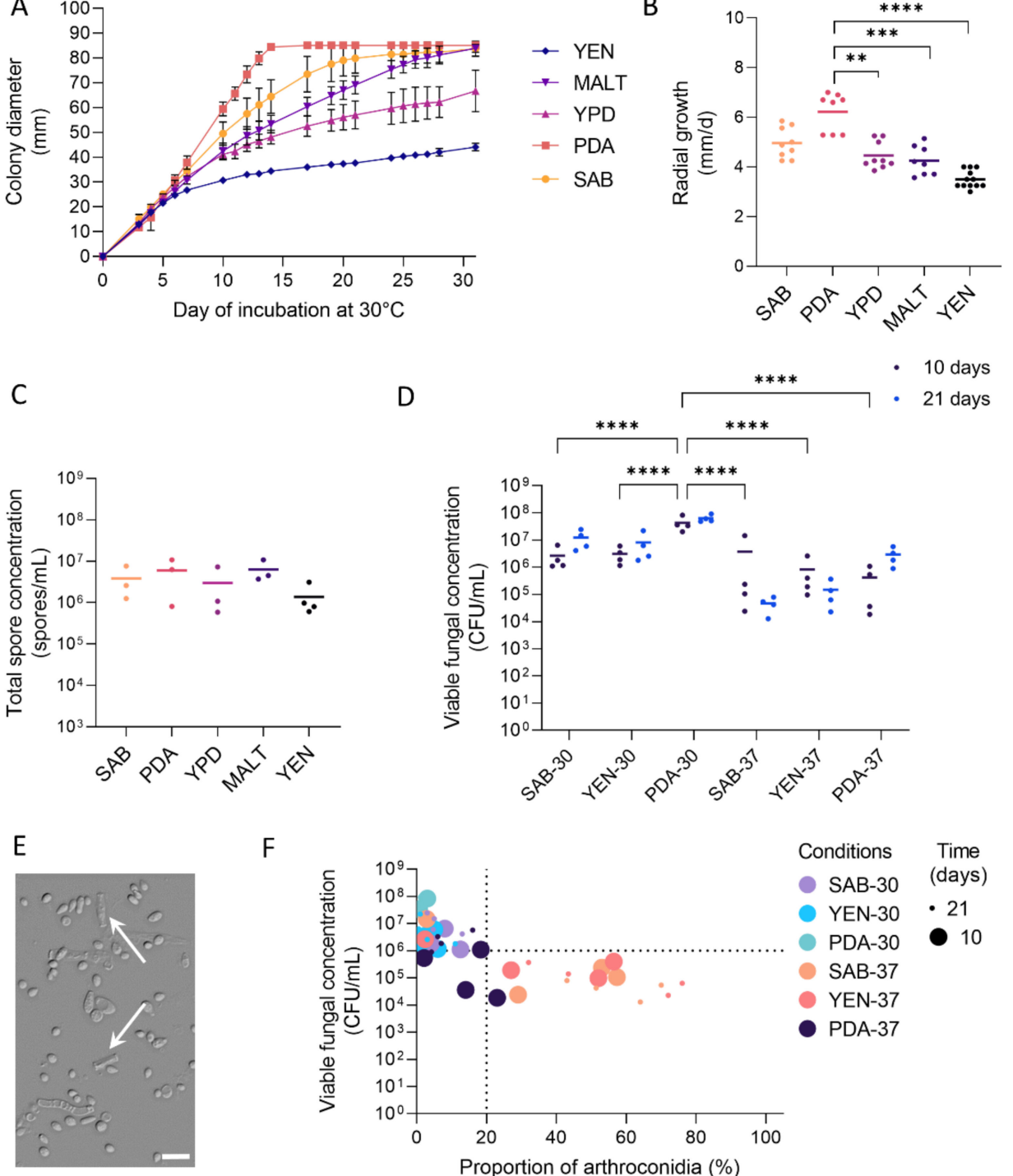

\section{F}
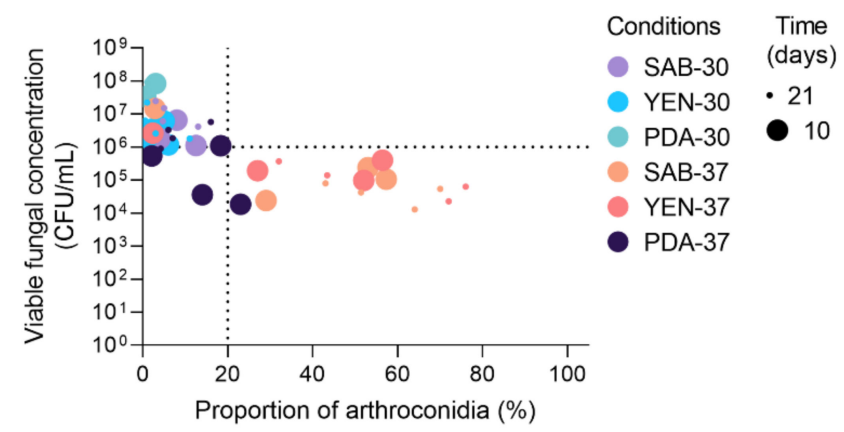

Figure 2. Growth and sporulation of T. benhamiae IHEM 20163. T. benhamiae IHEM 20163 were seeded over five agar media, Sabouraud (SAB), Potato Dextrose Agar (PDA), Yeast Peptone Dextrose (YPD), Malt medium (MALT) or Yeast Extract Nitrogen (YEN), and grown at $30{ }^{\circ} \mathrm{C}$ for 31 days. Fungal material was then scrapped, suspended in PBS and filtered to obtain fungal suspensions. (A) Diameter of fungal colonies was measured daily, (B) radial growth was determined during the exponential phase of growth, and (C) the total spore concentration recovered in fungal suspension was determined by counting using a Thoma chamber under light microscopy. To promote sporulation and arthroconidial production by T. benhamiae IHEM 20163, fungi were seeded on SAB, PDA or YEN media, and incubated at $30^{\circ} \mathrm{C}$ under $12 \% \mathrm{CO}_{2}$ or at $37^{\circ} \mathrm{C}$ under $10 \% \mathrm{CO}_{2}$, for 10 or 21 days. Fungal material was then scrapped, suspended in PBS, stirred and filtered to obtain spore suspensions. (D) Viable fungal concentration was determined by counting colony-forming units (CFU). (E) Morphology of arthroconidia (white arrow) was observed using differential interference contrast (DIC) microscopy. Scale bar $=10 \mu \mathrm{m}$. (F) Viable fungal concentration was correlated with the proportion of arthroconidia relative to the total number of fungal elements, determined by counting using a Thoma chamber under light microscopy. Dotted lines indicate the minimal viable fungal concentration required for further use on experimental models, based on the most important published models [15,16] and personal observations, as well as the proportion of $20 \%$ arthroconidia, arbitrarily chosen as a minimal value for suspensions enriched in arthroconidia. Data analysis: $n \geq 3$; means \pm SD for (A), means and individual values for (B-D), and single plots for (F); ANOVA2 for (B-D) compared to PDA; ${ }^{* *} p<0.01^{* * *} p<0.001^{* * * *} p<0.0001$. 
In parallel to growth, the sporulation of dermatophytes was assessed on each tested medium by determining the total spore concentration after 31 days of incubation, when the strains were in stationary phase on most media. Spores were mainly represented by microconidia and to a lesser extent by arthroconidia, with macroconidia being extremely rare due to the porosity of the Miracloth filter used for spore production (see Section 2.3 in Section 2). On PDA, the amount of spores produced by T. rubrum IHEM 13894 was significantly higher than on YPD, MALT, and YEN (Figure 1C). The same trend, whereas not statistically significant, was observed for T. benhamiae IHEM 20163 (Figure 2C). Consistently, PDA significantly increased the total spore concentration for M. canis IHEM 22957 compared to SAB, YPD, and YEN, and for T. mentagrophytes IHEM 25841 and TIMM 2789 compared to YEN (Figure S4). For all other strains, sporulation was higher on PDA than on the other media, but without significant difference.

\subsection{High Viable Fungal Concentration Is Obtained after Incubation on PDA}

In order to produce fungal suspensions suitable for use in experimental models, i.e., containing mainly unicellular spores (microconidia and ideally arthroconidia) with sufficient concentration and viability $\left(>10^{6} \mathrm{CFU} / \mathrm{mL}\right)$, dermatophytes were incubated for 10 or 21 days on $\mathrm{SAB}, \mathrm{YEN}$, and PDA at $30^{\circ} \mathrm{C}$ under $12 \% \mathrm{CO}_{2}$ or at $37^{\circ} \mathrm{C}$ under $10 \% \mathrm{CO}_{2}$. After the recovery of spore suspensions, the viable fungal concentration was evaluated by CFU counting.

For all the strains, except T. tonsurans IHEM 24958 and M. audouinii IHEM 10316, growth on PDA at $30{ }^{\circ} \mathrm{C}$ under $12 \% \mathrm{CO}_{2}$ resulted in sufficient quantity of viable fungal elements (FIgures 1D, 2D and S4-S6). Furthermore, for Trichophyton species, an incubation period of 10 days rather than 21 days did not have a significant negative impact on viable fungal concentration. For some strains, the variability of the fungal concentration obtained between replicates was even reduced, ultimately allowing a reduction in the culture time required. In contrast, for Microsporum species, a culture duration of less than 21 days appeared inappropriate due to lower viable fungal concentration.

For M. audouinii IHEM 10316 and T. tonsurans IHEM 24958, viable fungal concentration was extremely low, i.e., less than $10^{4} \mathrm{CFU} / \mathrm{mL}$, irrespective of conditions and media (Figures S5 and S7). Extending the incubation time to 31 days somehow increased the viable concentration, but it remained low and insufficient for further use in experiments.

\subsection{Production of Arthroconidia Is Highly Variable According to Strains and Culture Conditions}

Arthroconidia were observed using DIC microscopy and counted using a Thoma chamber under light microscopy to determine their percentage in the spore suspensions (see Section 2.4). In Microsporum species, the arthroconidia were rather rectangular in shape, with straight or curved edges, respectively for M. audouinii and M. canis (Figure S8). In contrast, their morphology was highly variable among Trichophyton species, with rectangular, spherical, or ovoid shapes (Figures 1E, 2E and S8). Furthermore, the morphology of arthroconidia may differ between their formation inside the hyphae and their release as individual cells, as for example arthroconidia of $T$. interdigitale appearing rectangular-shaped inside hyphae and spherical-shaped once they became individuals, while the contrary was observed for the arthroconidia of T. mentagrophytes (Figure S8).

The percentage of arthroconidia in spore suspensions varied considerably between culture conditions and between replicates for a given condition (Figures 1F, 2F, and S9). Furthermore, for each of the dermatophyte strains, the conditions favouring arthroconidial production, and those allowing sufficient viability $\left(>10^{6} \mathrm{CFU} / \mathrm{mL}\right)$ to be achieved, were not the same. Indeed, using a culture on PDA at $30^{\circ} \mathrm{C}$ under $12 \% \mathrm{CO}_{2}$ for 10 (Trichophyton spp.) or 21 days (Microsporum spp.), a condition defined as optimal in terms of viability, the proportion of arthroconidia was extremely variable and quite low $(<20 \%)$ for the majority of strains (Table 4, Figure S10). 
Table 4. Minimum and maximum proportions of arthroconidia produced after 10, 21 or 31 days of incubation on PDA at 30

${ }^{\circ} \mathrm{C}$ under $12 \% \mathrm{CO}_{2}$.

\begin{tabular}{|c|c|c|c|}
\hline \multirow{2}{*}{ Genus-Species- Isolate Number } & \multirow{2}{*}{ Incubation Duration (Days) } & \multicolumn{2}{|c|}{ Proportion of Arthroconidia (\%) } \\
\hline & & Min & $\operatorname{Max}$ \\
\hline \multicolumn{4}{|l|}{ Microsporum } \\
\hline \multirow{3}{*}{ M. audouinii IHEM 10316} & 10 & nd $^{1}$ & nd \\
\hline & 21 & nd & nd \\
\hline & 31 & nd & 67 \\
\hline M. canis IHEM 22957 & 21 & nd & 17 \\
\hline M. canis IHEM 22958 & 21 & 3 & 67 \\
\hline M. canis IHEM 21239 & 21 & 1 & 82 \\
\hline \multicolumn{4}{|l|}{ Trichophyton } \\
\hline T. benhamiae IHEM 20161 & 10 & 1 & 21 \\
\hline T. benhamiae IHEM 20163 & 10 & 1 & 1 \\
\hline T. interdigitale IHEM 00584 & 10 & 1 & 9 \\
\hline T. mentagrophytes IHEM 22733 & 10 & 14 & 24 \\
\hline T. mentagrophytes IHEM 22740 & 10 & 30 & 66 \\
\hline T. mentagrophytes IHEM 25841 & 10 & 1 & 15 \\
\hline T. mentagrophytes TIMM 2789 & 10 & 3 & 9 \\
\hline T. rubrum IHEM 13894 & 10 & 4 & 68 \\
\hline \multirow{3}{*}{ T. tonsurans IHEM 24958} & 10 & 60 & 100 \\
\hline & 21 & 29 & 100 \\
\hline & 31 & 72 & 93 \\
\hline
\end{tabular}

${ }^{1}$ A percentage below the limit of detection by Thoma chamber enumeration is indicated as "not determined" (nd).

3.4. Spore Suspensions Recovered after 10 Days on PDA at $30{ }^{\circ} \mathrm{C}$ under $12 \% \mathrm{CO}_{2}$ Are Adequate to Induce Infection on RHE

Mainly based on the viable fungal concentration, a 10-day (21-days for Microsporum species) incubation on PDA at $30{ }^{\circ} \mathrm{C}$ under $12 \% \mathrm{CO}_{2}$ appeared to be the optimal condition for the production of spore suspensions suitable as inoculum in experimental models. Indeed, in such conditions, the viable fungal concentration was above $10^{6} \mathrm{CFU} / \mathrm{mL}$, a concentration sufficient to induce infection when used in in vitro [15] and in vivo models [16]. Nevertheless, no information is available in the literature on the pathogenicity of spore suspensions of dermatophytes produced in such conditions. Thus, the infectious ability of these suspensions was evaluated in RHE model. Since the dermatophytosis model on RHE was initially developed using spore suspensions recovered after 21 days of incubation on YEN at $30{ }^{\circ} \mathrm{C}$ under $12 \% \mathrm{CO}_{2}$ as an inoculum [28], herein, we compared the infection development on RHE after topical application of spore suspensions recovered following the "conventional procedure" (21 days on YEN) or the "optimized procedure" (10 days on PDA). This comparison of the infection development was performed for the strains T. rubrum IHEM 13894 and T. benhamiae IHEM 20163. The size of inoculum was determined to induce a fungal invasion restricted to the cornified layer, as can be observed during natural in vivo infection, and appeared to be $1000 \mathrm{CFU}$ for T. rubrum and $30 \mathrm{CFU}$ for T. benhamiae [28].

\subsubsection{Adherence to RHE Is Improved for Spores Recovered on PDA}

As assessed by morphological analysis, invasion was restricted to the cornified layer, whatever the procedure used for inoculum production (Figures $3 \mathrm{~A}$ and $4 \mathrm{~A}$ ). Monitoring the fungal load of inoculum by seeding on SAB and subsequently performing CFU counting 
revealed a number of CFU close to the expected value (i.e., 30 CFU for T. benhamiae and 1000 CFU T. rubrum) (Figures 3B,C and 4B,C). In contrast, the percentage of adherent spores on the tissue surface after four hours of contact appeared significantly higher when the spores were produced on PDA in comparison with spores produced on YEN (Figures 3D and 4D). The more efficient adherence of spores produced on PDA resulted in a tendency towards a greater infection level quantified in the RHE three days or even more four days after their infection (Figures $3 \mathrm{E}$ and $4 \mathrm{E}$ ), as well as for a major impact on the functional integrity of the epidermal barrier (Figures $3 \mathrm{~F}$ and $4 \mathrm{~F}$ ).

A YEN PDA
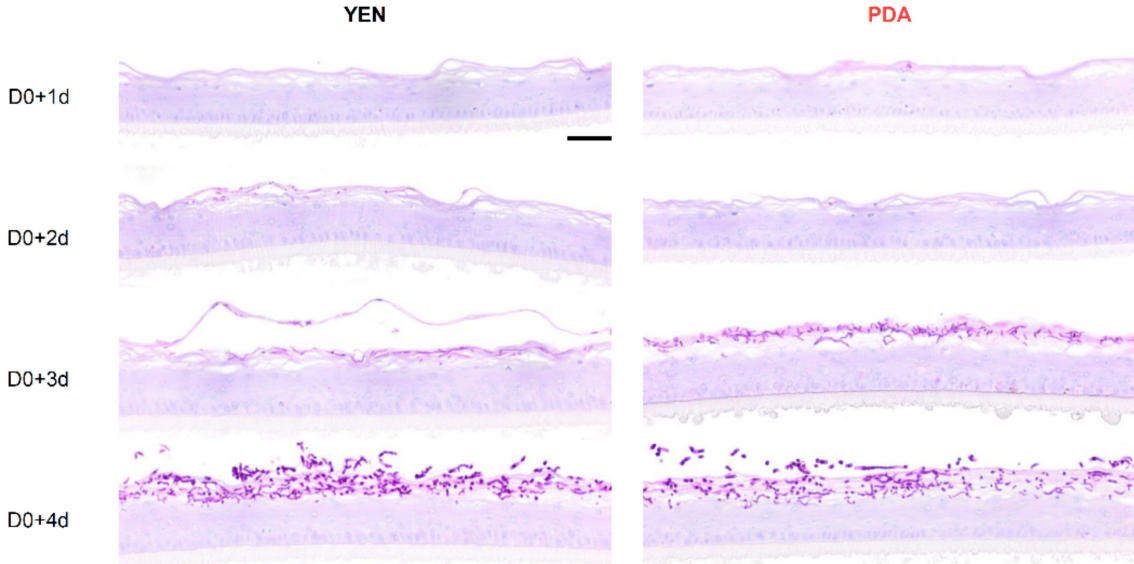

B

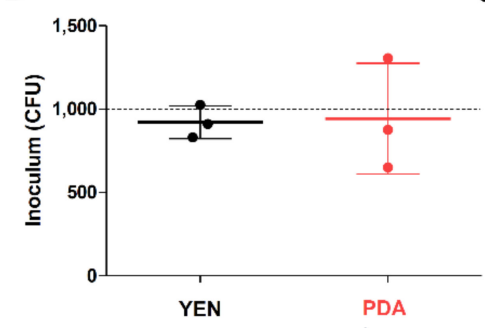

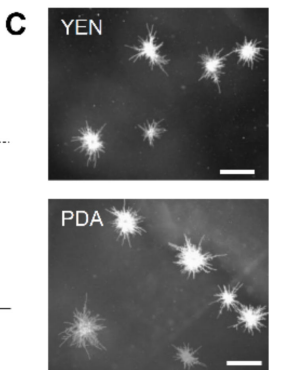

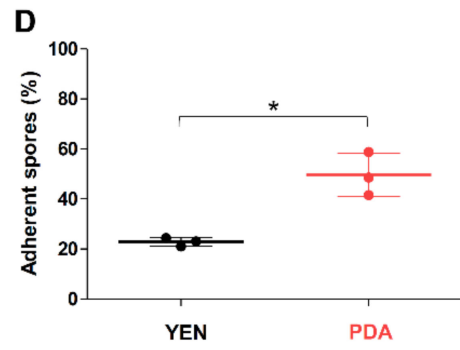

F

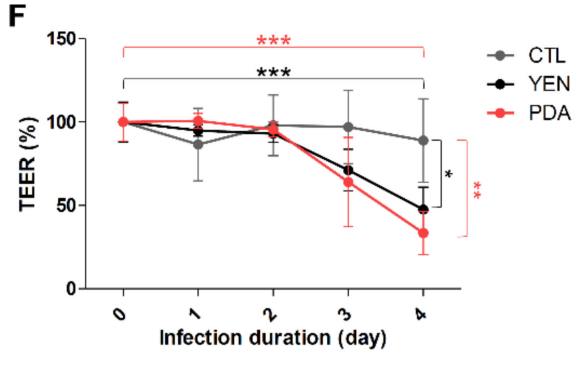

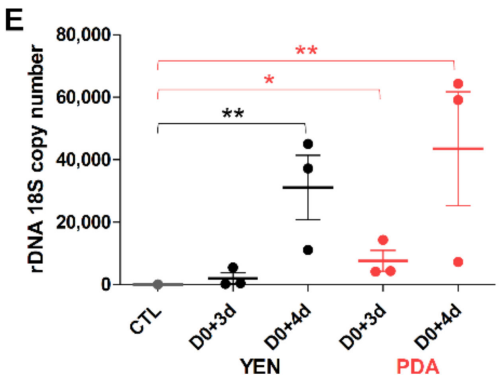

Figure 3. T. rubrum IHEM 13894 infection on reconstructed human epidermis (RHE). RHE were infected on day zero (D0) by topical addition of inoculum consisting in suspensions of T. rubrum IHEM 13894 spores recovered after 21 days of incubation over Yeast Extract Nitrogen agar (YEN) at $30{ }^{\circ} \mathrm{C}$ under $12 \% \mathrm{CO}_{2}$, or after 10 days of incubation over Potato Dextrose Agar (PDA) in the same conditions. The inoculum was adjusted to a density of 1000 colony-forming units (CFU) per RHE. (A) Histological sections were prepared from infected RHE analysed one (D0+1d), two (D0+2d), three $(\mathrm{D} 0+3 \mathrm{~d})$ or four $(\mathrm{D} 0+4 \mathrm{~d})$ days after infection and then stained using the Periodic-acid Schiff (PAS) procedure with $\alpha$-amylase pre-treatment and hemalun counterstaining. Scale bar $=50 \mu \mathrm{m}$. (B) Fungal load of inoculum was monitored by counting CFU, the dotted line indicates the expected size of inoculum, and (C) the macroscopic aspect of CFU was observed. Scale bars = $1 \mathrm{~mm}$. (D) Percentage of spores adhering to the epidermis was determined four hours after infection. (E) Infection levels were assessed three $(\mathrm{D} 0+3 \mathrm{~d})$ and four $(\mathrm{D} 0+4 \mathrm{~d})$ days after infection of RHE using qPCR analysis of the rDNA $18 \mathrm{~S}$ gene copy numbers of T. rubrum in total DNA extracts prepared from 
infected RHE (YEN and PDA) or non-infected RHE (CTL). (F) Epidermal barrier integrity of RHE during infection was determined by measurements of trans-epithelial electrical resistance (TEER). TEER values are expressed as percentage of values measured before RHE infection (D0). Data analysis: $\mathrm{n}=3$; means $\pm \mathrm{SD}$; ANOVA1 for $(\mathbf{B}, \mathbf{D})$, ANOVA2 for $(\mathbf{E}, \mathbf{F}){ }^{*} p<0.05^{* *} p<0.01^{* * *} p<0.001$.

A

YEN

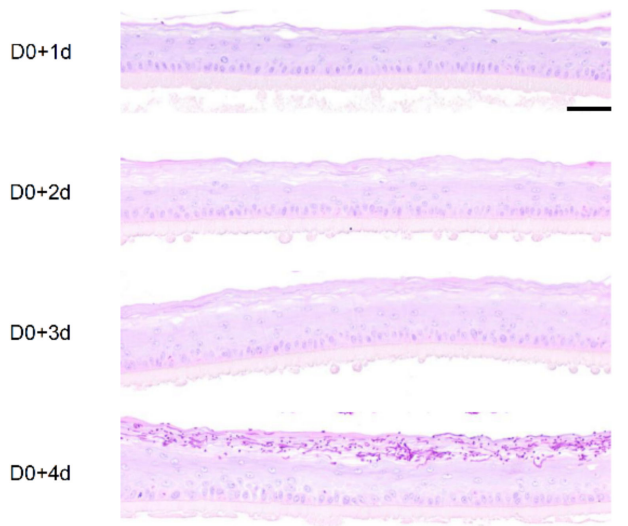

B

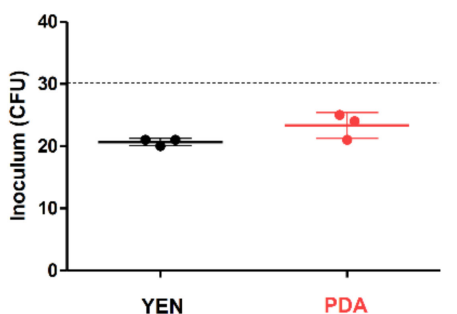

E

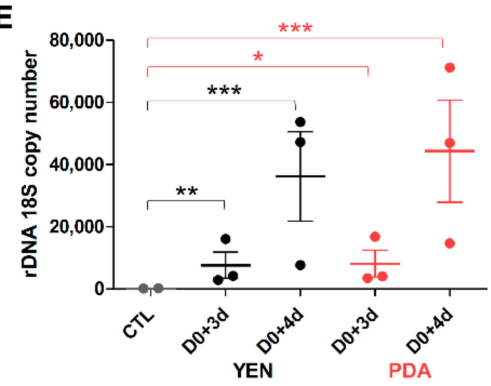

C YEN
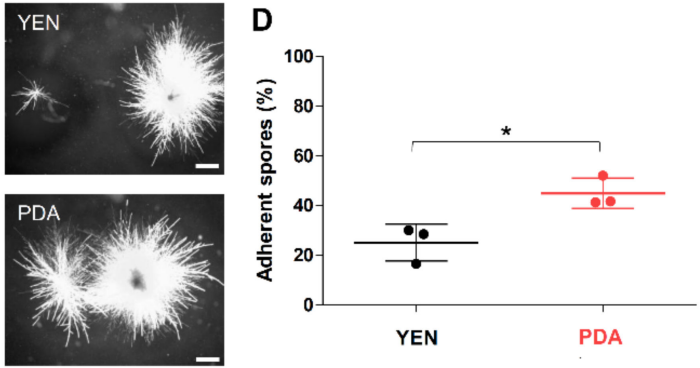

F

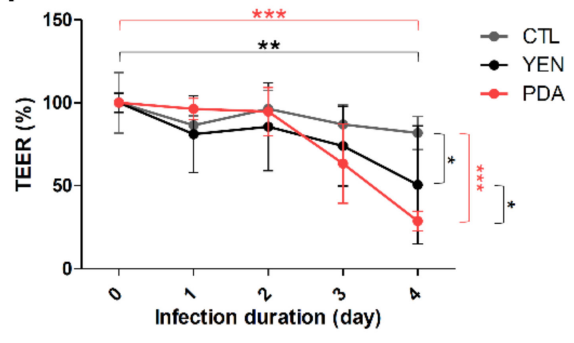

Figure 4. T. benhamiae IHEM 20163 infection on reconstructed human epidermis (RHE). RHE were infected on day zero (D0) by topical addition of inoculum consisting in suspensions of T. benhamiae IHEM 20163 spores recovered after 21 days of incubation over Yeast Extract Nitrogen agar (YEN) at $30{ }^{\circ} \mathrm{C}$ under $12 \% \mathrm{CO}_{2}$, or after 10 days of incubation over Potato Dextrose Agar (PDA) in the same conditions. The inoculum was adjusted to a density of 30 colony-forming units (CFU) per RHE. (A) Histological sections were prepared from infected RHE analysed one (D0+1d), two (D0+2d), three $(\mathrm{D} 0+3 \mathrm{~d})$ or four $(\mathrm{D} 0+4 \mathrm{~d})$ days after infection and then stained using the Periodic-acid Schiff (PAS) procedure with $\alpha$-amylase pre-treatment and hemalun counterstaining. Scale bar $=50 \mu \mathrm{m}$. (B) Fungal load of inoculum was monitored by counting CFU, the dotted line indicates the expected size of inoculum, and (C) the macroscopic aspect of CFU was observed. Scale bars $=1 \mathrm{~mm}$. (D) Percentage of spores adhering to the epidermis was determined four hours after infection. (E) Infection levels were assessed three (D0+3d) and four (D0+4d) days after infection of RHE using qPCR analysis of the rDNA $18 \mathrm{~S}$ gene copy numbers of T. benhamiae in total DNA extracts prepared from infected RHE (YEN and PDA) or non-infected RHE (CTL). (F) Epidermal barrier integrity of RHE during infection was determined by measurements of trans-epithelial electrical resistance (TEER). TEER values are expressed as percentage of 
values measured before RHE infection (D0). Data analysis: $\mathrm{n}=3$; means \pm SD; ANOVA1 for (B,D), ANOVA2 for $(\mathbf{E}, \mathbf{F}){ }^{*} p<0.05^{* *} p<0.01^{* * *} p<0.001$.

3.4.2. Responses of Keratinocytes to Infection Are More Consistent When Spores Are Produced on PDA

Keratinocyte responses to the infection of RHE by dermatophytes were assessed through measurements of mRNA expression and release in the culture medium of several pro-inflammatory cytokines (IL-1 $\alpha$, IL-1 $\beta$, IL- 8 and TNF $\alpha$ ) and antimicrobial peptides ( $\beta$-defensin-2 (BD2) and -3 (BD3) and cathelicidin LL-37) (Figures 5, 6 and S11). Since a previous report from our team has shown that keratinocyte responses mainly appear near the end of the infection period [29], the expression and release of these factors were monitored on the third and fourth days following infection of the RHE. In accordance with previously obtained results, keratinocytes expressed and released proinflammatory cytokines and antimicrobial peptides mainly on the fourth day following infection, and at similar level whatever the procedure used for spore production, either on YEN or on PDA. However, the variability of keratinocyte responses using the inoculum produced on YEN was very high between replicate assays. This factual observation that has already been reported [29] remains difficult to explain and monitor. In contrast, the reproducibility of the observed keratinocyte responses was improved and quite satisfactory with the inoculum produced on PDA.

A

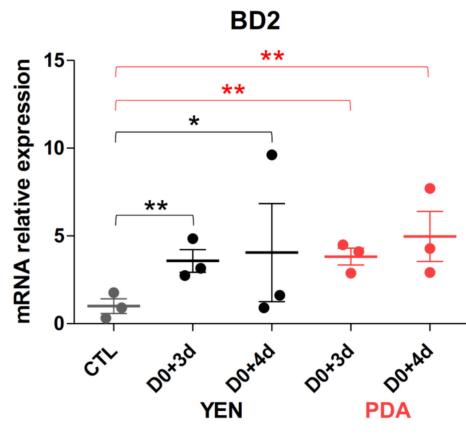

IL-1 $\alpha$
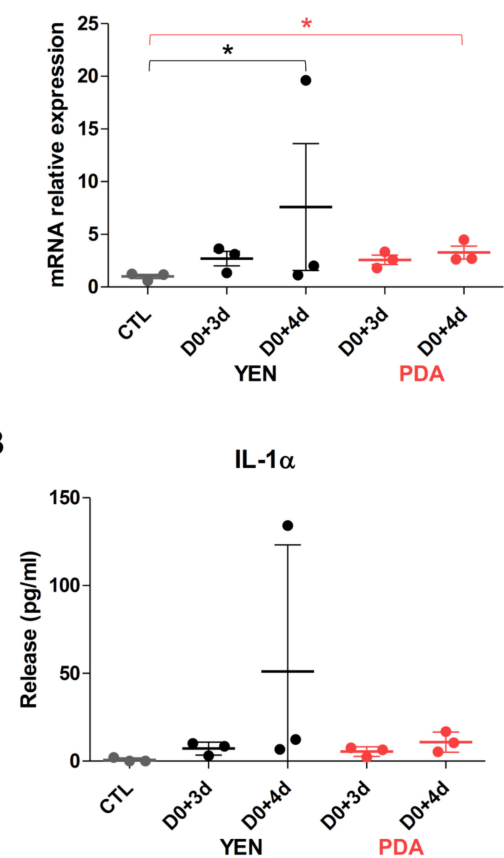

BD3

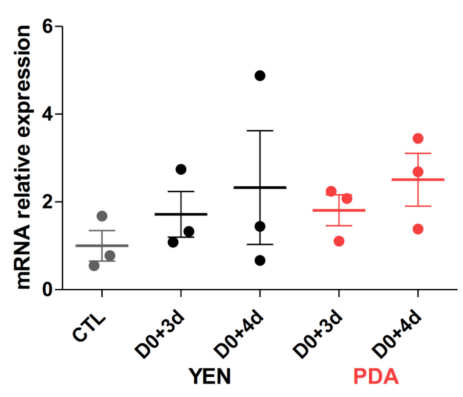

IL-8

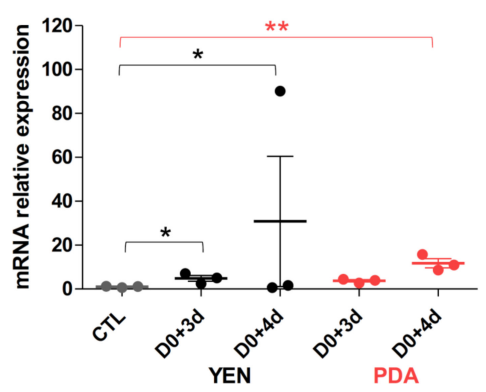

IL-8

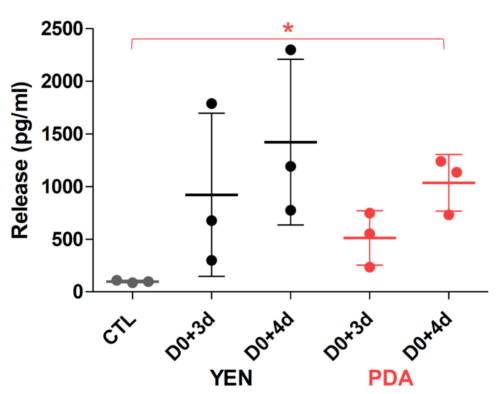

Figure 5. Keratinocytes responses during T. rubrum IHEM 13894 infection on reconstructed human 
epidermis (RHE). RHE were infected on day zero (D0) by topical addition of inoculum consisting in suspensions of T. rubrum IHEM 13894 spores recovered after 21 days of incubation over Yeast Extract Nitrogen agar (YEN) at $30{ }^{\circ} \mathrm{C}$ under $12 \% \mathrm{CO}_{2}$, or after 10 days of incubation over Potato Dextrose Agar (PDA) in the same conditions. The inoculum has been adjusted in each case to a density of 1000 colony-forming units (CFU) per RHE. (A) mRNA expression of antimicrobial peptides (BD2, BD3) and pro-inflammatory cytokines (IL- $1 \alpha$, IL- 8 ) and (B) release of IL- $1 \alpha$ and IL- 8 by infected RHE, three (D0+3d) and four (D0+4d) days after infection, or by non-infected RHE (CTL) were assessed respectively by RT-qPCR and ELISA. Data analysis: $\mathrm{n}=3$; means $\pm \mathrm{SD}$; ANOVA2; ${ }^{*} p<0.05{ }^{* *} p<0.01$.

A
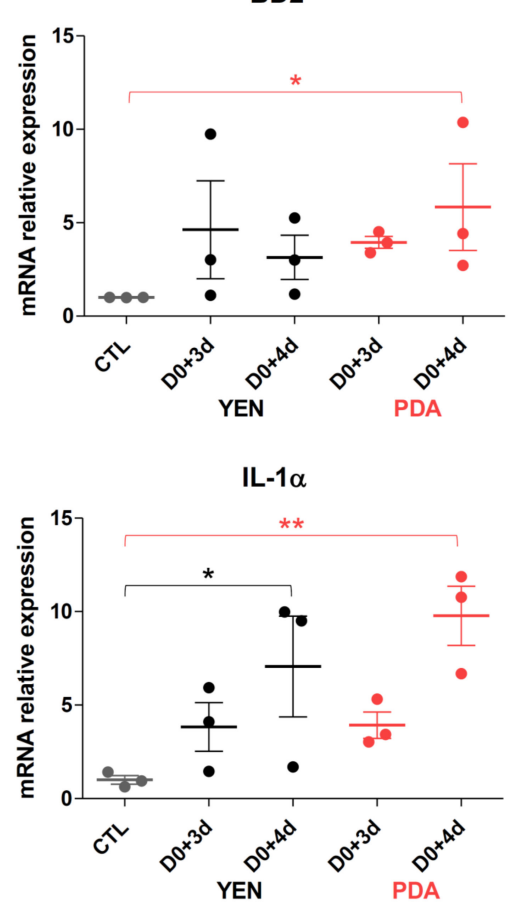

B

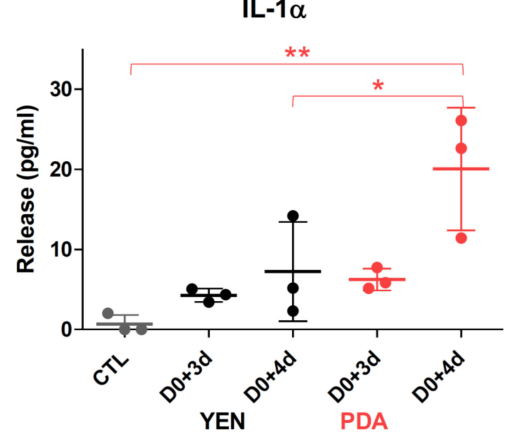

BD3

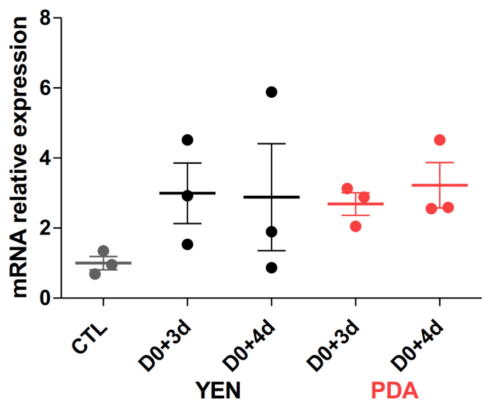

IL-8

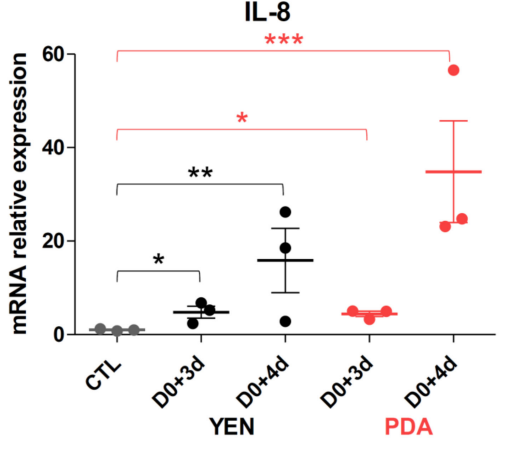

IL-8

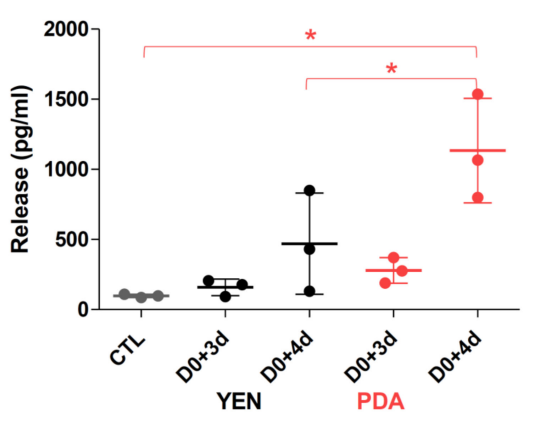

Figure 6. Keratinocytes responses during T. benhamiae IHEM 20163 infection on reconstructed human epidermis (RHE). RHE were infected on day zero (D0) by topical addition of inoculum consisting in suspensions of T. benhamiae IHEM 20163 spores recovered after 21 days of incubation over Yeast Extract Nitrogen agar (YEN) at $30{ }^{\circ} \mathrm{C}$ under $12 \% \mathrm{CO}_{2}$, or after 10 days of incubation over Potato Dextrose Agar (PDA) in the same conditions. The inoculum has been adjusted in each case to a density of 30 colony-forming units (CFU) per RHE. (A) mRNA expression of antimicrobial peptides (BD2, BD3) and pro-inflammatory cytokines (IL- $1 \alpha$, IL-8) and (B) release of IL- $1 \alpha$ and IL- 8 by infected RHE, three (D0+3d) and four (D0+4d) days after infection, or by non-infected RHE (CTL) were assessed respectively by RT-qPCR and ELISA. Data analysis: $\mathrm{n}=3$; means \pm SD; ANOVA2; ${ }^{*} p<0.05^{* *} p<0.01^{* * *} p<0.001$. 


\section{Discussion}

Faced with a still increasing incidence of dermatophytosis [2], together with the emergence of antifungal resistance [10-14], a better understanding of fungal mechanisms involved in the pathogenesis of dermatophytosis, as well as of cellular responses developed by the host, has become crucially awaited in order to rationally define new therapeutic strategies. For this purpose, several in vitro and in vivo infection models have been developed, allowing actual advances in this field [49]. However, unwanted heterogeneity in the production and composition of inocula renders difficult the overall interpretation of observations made on these various models. With the aim to develop some standardized and reproducible procedures for the production of infective spores suitable as inoculum in models, this study characterized several dermatophyte species frequently isolated from human lesions for their growth rate, sporulation, and arthroconidial production in different culture conditions.

Among the five tested media, i.e., SAB, PDA, YPD, MALT, and YEN depicted as classical media for fungal growth, PDA appeared to improve the growth and sporulation of most dermatophyte strains (e.g., T. benhamiae strains and T. mentagrophytes TIMM 2789). For other strains, there was no or little differences between media, especially between PDA and SAB, which also efficiently allowed dermatophyte growth and sporulation (e.g., T. rubrum IHEM 13894 and T. interdigitale IHEM 00584). Furthermore, the growth of Microsporum species, and particularly M. canis IHEM 21239, was slightly increased on YEN, in favour of the previous use of this medium to produce fungal suspensions of $M$. canis for infection in in vitro [25] and in vivo [26,27] models. However, in order to standardize the medium used for all species and strains, the PDA appears more appropriate.

In accordance with the better fungal growth on PDA, spore suspensions produced from this medium showed a high viable fungal concentration $\left(>10^{6} \mathrm{CFU} / \mathrm{mL}\right)$, sufficient for further use in in vitro and in vivo infection models, as based on the most important published models $[15,16]$ and personal observations. Addition of the stirring step before the filtration during the procedure of recovery increased the viable fungal concentration by allowing a better separation of unicellular spores from hyphae. This stirring step can be performed through vortexing the suspension for one minute ("fast procedure" used in this paper), or by a four-hours shaking at $4{ }^{\circ} \mathrm{C}$ ("long procedure") for an even more efficient separation of spores, and thereby a higher viable fungal concentration. Furthermore, a shorter incubation time on PDA, 10 days instead of 21, did not have a negative impact on the viable fungal concentration for Trichophyton species, which is an advantage in routine laboratory production.

M. audouinii IHEM 10316 and T. tonsurans IHEM 25841 are exceptions, as since very low viable fungal concentrations, of less than $10^{4}$ and $10^{5} \mathrm{CFU} / \mathrm{mL}$ respectively, were obtained for both strains, irrespective of the medium and conditions used, and even after a prolonged incubation of 31 days. Interestingly, the total spore concentration for both these strains appeared to be higher under atmospheric tension compared to incubation under $10 \% \mathrm{CO}_{2}$ or $12 \% \mathrm{CO}_{2}$, suggesting that incubation of these strains under lower $\mathrm{CO}_{2}$ tension should be an alternative to recover spores to a concentration compatible with their use in infectious models.

Dermatophytes are able to produce two types of spores: micro- and macroconidia resulting from hyphal budding, or arthroconidia derived from hyphal fragmentation. Although arthroconidia are described as natural vectors of dermatophytes [36], they have been less frequently used in experimental models because they remain difficult to produce in culture. Indeed, irregular successes have been described for an efficient in vitro production of arthroconidia [39,40,50-52]. Based on previous studies reporting the production of arthroconidia after 10 - or 14-day incubation on $\mathrm{SAB}$ at $37^{\circ} \mathrm{C}$ under $10 \% \mathrm{CO}_{2}[43,44,52]$, or after 14-21-day incubation on YEN at $30{ }^{\circ} \mathrm{C}$ under $12 \% \mathrm{CO}_{2}$ [25], and on our observation that PDA enhances sporulation, we compared the production of arthroconidia by dermatophytes after 10 or 21 days of incubation on SAB, YEN, or PDA, at $37^{\circ} \mathrm{C}$ under $10 \% \mathrm{CO}_{2}$ or at $30{ }^{\circ} \mathrm{C}$ under $12 \% \mathrm{CO}_{2}$. Testing these conditions is in agreement with previous reports 
showing that PDA medium and high $\mathrm{CO}_{2}$ tension are factors that improve dermatophyte sporulation and arthroconidial production [50,53].

In the literature, arthroconidia are described as short-chained or spherical or oval unicellular spores $[37,54]$ and are characterized by the presence of a thicker cell wall when compared to microconidia [55]. In our experience, the morphology of arthroconidia appears highly variable and their identification requires an expert observer, which reinforces the need for molecular tools able to facilitate standardized routine identification of arthroconidia. The proportion of arthroconidia numbered in fungal suspensions recovered after incubation on SAB, PDA and YEN was also highly variable between dermatophytes strains, as well as between the culture medium and condition used, and between replicate assays. In addition, the conditions that enhanced arthroconidial production were not the same as those that allowed a high viable concentration required for subsequent infection in experimental models. Nevertheless, after incubation on PDA at $30{ }^{\circ} \mathrm{C}$ under $12 \% \mathrm{CO}_{2}$, identified as the optimal culture condition for viable fungal concentration, dermatophytes produce arthroconidia with percentages ranging from 1\% to 68\% for T. rubrum IHEM 13894 or $82 \%$ for M. canis IHEM 21239. The spore suspensions produced in these conditions are therefore "enriched in arthroconidia" compared to suspensions exclusively composed of microconidia and previously used as inoculum in many models [17-23].

Finally, to ensure that spores produced using PDA at $30{ }^{\circ} \mathrm{C}$ under $12 \% \mathrm{CO}_{2}$ are able to adhere to and invade host tissue, and thus constitute an adequate inoculum, we evaluated the development of infection by the anthropophilic T. rubrum IHEM 13894 and the zoophilic T. benhamiae IHEM 20163 strains using the pre-established model of dermatophytosis on RHE [28]. Spores produced on PDA were able to invade the tissue, leading to tissue damages through impairment of the epidermal barrier, and inducing responses by keratinocytes, as measured by the expression and release of cytokines and antimicrobial peptides. Strangely, the adherence of spores to the RHE surface after four hours of contact appeared to be increased when the inoculum was produced on PDA compared to the inoculum produced by the conventional method used for the design of the model (i.e., 21 days of incubation on YEN [28]). In addition, the measured responses of keratinocytes, which were pretty variable when spores were produced on YEN, were more reproducible with spores produced on PDA.

In conclusion, this study proposes a standardized procedure for the production of infective spores for use as routine inoculum in experimental models of dermatophytosis. This procedure, summarized in Table 5, allows the recovery of fungal suspensions made of unicellular spores, microconidia but also arthroconidia, at a viable concentration sufficient for infection in in vitro and in vivo models. Standardization of the inocula used in the different experimental models developed by research teams worldwide will facilitate reproducibility and the comparison of observations, leading to more uniform results, which will ultimately improve knowledge of the pathogenesis of dermatophytosis.

Table 5. Recommendations for a standardized procedure for the production of infective spores useable as inoculum in in vitro and in vivo experimental models.

\begin{tabular}{|c|c|c|c|}
\hline Step & Procedure & Aim & Comment \\
\hline Preculture & Incubation for 14 days on $\mathrm{SAB}$ at $30^{\circ} \mathrm{C}$ & Growth of the starting fungal material & \\
\hline \multicolumn{4}{|c|}{ Recovering of fungal material by scraping and suspension in PBS } \\
\hline Sporulation & $\begin{array}{l}\text { Seeding on PDA and incubation for } 10 \\
\text { days at } 30{ }^{\circ} \mathrm{C} \text { under } 12 \% \mathrm{CO}_{2}\end{array}$ & $\begin{array}{l}\text { Production of unicellular spores, } \\
\text { mainly microconidia, and to a lesser } \\
\text { extent arthroconidia }\end{array}$ & $\begin{array}{l}21 \text { days required for } \\
\text { Microsporum spp. }\end{array}$ \\
\hline \multicolumn{4}{|c|}{ Recovering of fungal material by scraping and suspension in PBS } \\
\hline Agitation & Four hours at $4{ }^{\circ} \mathrm{C}$ using magnetic stirrer & $\begin{array}{l}\text { Separation of unicellular spores } \\
\text { from hyphae }\end{array}$ & $\begin{array}{l}\text { One-minute vortexing for } \\
\text { fast procedure (reduced } \\
\text { efficiency) }\end{array}$ \\
\hline
\end{tabular}


Table 5. Cont.

\begin{tabular}{|c|c|c|c|}
\hline Filtration & Through three Miracloth layers & Recovery of unicellular elements only & $\begin{array}{l}\text { Short hyphae can sometimes } \\
\text { pass through the } \\
\text { Miracloth filter }\end{array}$ \\
\hline Washing & $\begin{array}{l}\text { Centrifugation of the spore suspension } \\
\text { at } 3000 \times g \text { for } 5 \text { min, discarding of the } \\
\text { supernatant and resuspension of the } \\
\text { pellet in PBS }\end{array}$ & Elimination of medium residues & $\begin{array}{l}\text { Repeat until obtention of a } \\
\text { clear supernatant, and at } \\
\text { least three times }\end{array}$ \\
\hline Final suspension & $\begin{array}{l}\text { Resuspension of the pellet in an } \\
\text { appropriate volume of PBS depending } \\
\text { of the size of the pellet and of the final } \\
\text { spore concentration wanted }\end{array}$ & & $\begin{array}{l}\text { Store spore suspensions at } 4 \\
{ }^{\circ} \mathrm{C} \text { and use within one month }\end{array}$ \\
\hline
\end{tabular}

Supplementary Materials: The following are available online at https:/ / www.mdpi.com/article / 10.3390/jof7121029/s1, Figure S1: Impact of the culture medium on fungal growth, Figure S2: Impact of the culture medium on the radial growth of dermatophytes, Figure S3: Macroscopic aspect of T. rubrum IHEM 13894 and of T. benhamiae IHEM 20163 colonies, Figure S4: Impact of medium on sporulation of dermatophytes, Figure S5: Viable fungal concentration in infective spore suspensions of Trichophyton species, Figure S6: Viable fungal concentration in infective spore suspensions of Trichophyton mentagrophytes strains, Figure S7: Viable fungal concentration in infective spore suspensions of Microsporum species, Figure S8: Morphology of dermatophyte arthroconidia, Figure S9: Correlation between viable fungal concentration and percentage of arthroconidia in spore suspensions recovered under several culture conditions, Figure S10: Microscopic aspect of spore suspensions of T. rubrum IHEM 13894 and of T. benhamiae IHEM 20163, Figure S11: Keratinocyte responses during (A) T. rubrum IHEM 13894 or (B) T. benhamiae IHEM 20163 infection on reconstructed human epidermis (RHE).

Author Contributions: Conceptualization, E.F., C.S., B.M., and Y.P.; methodology, E.F., C.S., B.M., and Y.P.; validation, E.F., C.S., B.M., and Y.P.; formal analysis, E.F. and C.S.; investigation, E.F., C.S., S.V., C.D., and C.C.; data curation, E.F. and C.S.; writing-original draft preparation, E.F., C.S., B.M., and Y.P.; writing-review and editing, E.F., C.S., B.M., and Y.P.; visualization, E.F. and C.S.; supervision, E.F., C.S., B.M., and Y.P.; project administration, E.F. and C.S.; funding acquisition, E.F., B.M., and Y.P. All authors have read and agreed to the published version of the manuscript.

Funding: This research was funded by the Région Wallonne, MYCEPI grant number 1910074.

Institutional Review Board Statement: Not applicable.

Informed Consent Statement: Not applicable.

Acknowledgments: The authors gratefully acknowledge the technical help provided by V. Bielarz, V. De Glas and F. Marechal. Special thanks to B. Bienfait and JS. Blairvacq for providing human skin samples, and to T. Yamada, from the Institute of Medical Mycology, Teikyo University, for providing the strain T. mentagrophytes TIMM 2789. Special thanks are addressed to M. Salmon and C. Lancelot from the StratiCELL company, Les Isnes, Belgium, for their valuable advice and financial support through the MYCEPI grant.

Conflicts of Interest: The authors declare no conflict of interest.

\section{References}

1. Weitzman, I.; Summerbell, R.C. The dermatophytes. Clin. Microbiol. Rev. 1995, 8, 240-259. [CrossRef]

2. Havlickova, B.; Czaika, V.A.; Friedrich, M. Epidemiological trends in skin mycoses worldwide. Mycoses 2008, 51, 2-15. [CrossRef] [PubMed]

3. Saunte, D.M.; Holgersen, J.B.; Haedersdal, M.; Strauss, G.; Bitsch, M.; Svendsen, O.L.; Arendrup, M.C.; Svejgaard, E.L. Prevalence of toe nail onychomycosis in diabetic patients. Acta Derm. Venereol. 2006, 86, 425-428. [CrossRef]

4. Gupta, A.K.; Daigle, D.; Foley, K.A. The prevalence of culture-confirmed toenail onychomycosis in at-risk patient populations. J. Eur. Acad. Dermatol. Venereol. 2015, 29, 1039-1044. [CrossRef] [PubMed]

5. Eckhard, M.; Lengler, A.; Liersch, J.; Bretzel, R.G.; Mayser, P. Fungal foot infections in patients with diabetes mellitus-Results of two independent investigations. Mycoses 2007, 50, 14-19. [CrossRef] [PubMed] 
6. Sacheli, R.; Cuypers, L.; Seidel, L.; Darfouf, R.; Adjetey, C.; Lagrou, K.; Hayette, M.P. Epidemiology of dermatophytes in Belgium: A 5 years' survey. Mycopathologia 2021, 186, 399-409. [CrossRef] [PubMed]

7. Gnat, S.; Lagowski, D.; Nowakiewicz, A. Genetic predisposition and its heredity in the context of increased prevalence of dermatophytoses. Mycopathologia 2021, 186, 163-176. [CrossRef]

8. Gupta, A.K.; Foley, K.A.; Versteeg, S.G. New antifungal agents and new formulations against dermatophytes. Mycopathologia 2016, 182, 127-141. [CrossRef]

9. Raschi, E.; Poluzzi, E.; Koci, A.; Caraceni, P.; Ponti, F.D. Assessing liver injury associated with antimycotics: Concise literature review and clues from data mining of the FAERS database. World J. Hepatol. 2014, 6, 601-612. [CrossRef] [PubMed]

10. Monod, M.; Feuermann, M.; Salamin, K.; Fratti, M.; Makino, M.; Alshahni, M.M.; Makimura, K.; Yamada, T. Trichophyton rubrum azole resistance mediated by a new ABC transporter, TruMDR3. Antimicrob. Agents Chemother. 2019, 63, e00863-19. [CrossRef] [PubMed]

11. Lagowski, D.; Gnat, S.; Nowakiewicz, A.; Osinska, M.; Dylag, M. Intrinsic resistance to terbinafine among human and animal isolates of Trichophyton mentagrophytes related to amino acid substitution in the squalene epoxidase. Infection 2020, 48, 889-897. [CrossRef]

12. Kakurai, M.; Harada, K.; Maeda, T.; Hiruma, J.; Kano, R.; Demitsu, T. Case of tinea corporis due to terbinafine-resistant Trichophyton interdigitale. J. Dermatol. 2020, 47, e104-e105. [CrossRef]

13. Gu, D.; Hatch, M.; Ghannoum, M.; Elewski, B.E. Treatment-resistant dermatophytosis: A representative case highlighting an emerging public health threat. JAAD Case Rep. 2020, 6, 1153-1155. [CrossRef] [PubMed]

14. Gaurav, V.; Bhattacharya, S.N.; Sharma, N.; Datt, S.; Kumar, P.; Rai, G.; Singh, P.K.; Taneja, B.; Das, S. Terbinafine resistance in dermatophytes: Time to revisit alternate antifungal therapy. J. Mycol. Med. 2021, 31, 101087. [CrossRef]

15. Faway, E.; Lambert de Rouvroit, C.; Poumay, Y. In vitro models of dermatophyte infection to investigate epidermal barrier alterations. Exp. Dermatol. 2018, 27, 915-922. [CrossRef] [PubMed]

16. Cambier, L.; Heinen, M.P.; Mignon, B. Relevant animal models in dermatophyte research. Mycopathologia 2017, 182, 229-240. [CrossRef]

17. Achterman, R.R.; Moyes, D.L.; Thavaraj, S.; Smith, A.R.; Blair, K.M.; White, T.C.; Naglik, J.R. Dermatophytes activate skin keratinocytes via mitogen-activated protein kinase signaling and induce immune responses. Infect. Immun. 2015, 83, 1705-1714. [CrossRef]

18. Liang, P.; Huang, X.; Yi, J.; Chen, Z.; Ma, H.; Ye, C.; Chen, X.; Lai, W.; Chen, J. Trichophyton rubrum infection model based on the reconstructed human epidermis-Episkin. Chin. Med. J. 2016, 129, 54-58. [CrossRef] [PubMed]

19. Shi, Y.; Niu, Q.; Yu, X.; Jia, X.; Wang, J.; Lin, D.; Jin, Y. Assessment of the function of SUB6 in the pathogenic dermatophyte Trichophyton mentagrophytes. Med. Mycol. 2016, 54, 59-71.

20. Burstein, V.L.; Guasconi, L.; Beccacece, I.; Theumer, M.G.; Mena, C.; Prinz, I.; Cervi, L.; Herrero, M.; Masih, D.T.; Chiapello, L.S. IL-17-mediated immunity controls skin infection and T Helper 1 response during experimental Microsporum canis dermatophytosis. J. Investig. Dermatol. 2018, 138, 1744-1753. [CrossRef]

21. Heinen, M.P.; Cambier, L.; Antoine, N.; Gabriel, A.; Gillet, L.; Bureau, F.; Mignon, B. Th1 and Th17 immune responses act complementarily to optimally control superficial dermatophytosis. J. Investig. Dermatol. 2019, 139, 626-637. [CrossRef]

22. Baumbach, C.M.; Michler, J.K.; Nenoff, P.; Uhrlass, S.; Schrodl, W. Visualising virulence factors: Trichophyton benhamiae subtilisins demonstrated in a guinea pig skin ex vivo model. Mycoses 2020, 63, 970-978. [CrossRef]

23. Baumbach, C.M.; Schrodl, W.; Nenoff, P.; Uhrlass, S.; Mulling, C.K.W.; Michler, J.K. Modeling dermatophytosis: Guinea pig skin explants represent a highly suitable model to study Trichophyton benhamiae infections. J. Dermatol. 2020, 47, 8-16. [CrossRef] [PubMed]

24. Fujita, S.; Matsuyama, T. Experimental tinea pedis induced by non-abrasive inoculation of Trichophyton mentagrophytes arthrospores on the plantar part of a guinea pig foot. J. Med. Vet. Mycol. 1987, 25, 203-213. [CrossRef]

25. Tabart, J.; Baldo, A.; Vermout, S.; Nusgens, B.; Lapiere, C.; Losson, B.; Mignon, B. Reconstructed interfollicular feline epidermis as a model for Microsporum canis dermatophytosis. J. Med. Microbiol. 2007, 56, 971-975. [CrossRef]

26. Baldo, A.; Mathy, A.; Tabart, J.; Camponova, P.; Vermout, S.; Massart, L.; Marechal, F.; Galleni, M.; Mignon, B. Secreted subtilisin Sub3 from Microsporum canis is required for adherence to but not for invasion of the epidermis. Br. J. Dermatol. 2010, 162, 990-997. [CrossRef]

27. Cambier, L.; Bagut, E.T.; Heinen, M.P.; Tabart, J.; Antoine, N.; Mignon, B. Assessment of immunogenicity and protective efficacy of Microsporum canis secreted components coupled to monophosphoryl lipid-A adjuvant in a vaccine study using guinea pigs. Vet. Microbiol. 2015, 175, 304-311. [CrossRef]

28. Faway, E.; Cambier, L.; Mignon, B.; Poumay, Y.; Lambert de Rouvroit, C. Modeling dermatophytosis in reconstructed human epidermis: A new tool to study infection mechanisms and to test antifungal agents. Med. Mycol. 2017, 55, 485-494. [CrossRef] [PubMed]

29. Faway, E.; Cambier, L.; De Vuyst, E.; Evrard, C.; Thiry, M.; Lambert de Rouvroit, C.; Mignon, B.; Poumay, Y. Responses of reconstructed human epidermis to Trichophyton rubrum infection and impairment of infection by the inhibitor PD169316. J. Investig. Dermatol. 2019, 139, 2080-2089. [CrossRef]

30. Odds, F.; Ausma, J.; Van Gerven, F.; Woestenborghs, F.; Meerpoel, L.; Heeres, J.; Vanden Bossche, H.; Borgers, M. In vitro and in vivo activities of the novel antifungal agent r126638. Antimicrob. Agents Chemother. 2004, 48, 388-391. [CrossRef] [PubMed] 
31. Staib, P.; Zaugg, C.; Mignon, B.; Weber, J.; Grumbt, M.; Pradervand, S.; Harshman, K.; Monod, M. Differential gene expression in the pathogenic dermatophyte Arthroderma benhamiae in vitro versus during infection. Microbiology 2010, 156, 884-895. [CrossRef]

32. Grumbt, M.; Defaweux, V.; Mignon, B.; Monod, M.; Burmester, A.; Wostemeyer, J.; Staib, P. Targeted gene deletion and in vivo analysis of putative virulence gene function in the pathogenic dermatophyte Arthroderma benhamiae. Eukaryot. Cell 2011, 10, 842-853. [CrossRef] [PubMed]

33. Cambier, L.; Weatherspoon, A.; Defaweux, V.; Bagut, E.T.; Heinen, M.P.; Antoine, N.; Mignon, B. Assessment of the cutaneous immune response during Arthroderma benhamiae and A. vanbreuseghemii infection using an experimental mouse model. Br. J. Dermatol. 2014, 170, 625-633. [CrossRef]

34. Singh, G.; Kumar, P.; Joshi, S.C. Treatment of dermatophytosis by a new antifungal agent 'apigenin'. Mycoses 2014, 57, 497-506. [CrossRef]

35. Tran, V.D.; De Coi, N.; Feuermann, M.; Schmid-Siegert, E.; Bagut, E.T.; Mignon, B.; Waridel, P.; Peter, C.; Pradervand, S.; Pagni, M.; et al. RNA sequencing-based genome reannotation of the dermatophyte Arthroderma benhamiae and characterization of its secretome and whole gene expression profile during infection. mSystems 2016, 1, 00036-16. [CrossRef]

36. Rashid, A. Arthroconidia as vectors of dermatophytosis. Cutis 2001, 67, 23. [PubMed]

37. Hashimoto, T.; Blumenthal, H.J. Factors affecting germination of Trichophyton mentagrophytes arthrospores. Infect. Immun. 1977, 18, 479-486. [CrossRef]

38. Hoff, B.; Schmitt, E.K.; Kuck, U. CPCR1, but not its interacting transcription factor AcFKH1, controls fungal arthrospore formation in Acremonium chrysogenum. Mol. Microbiol. 2005, 56, 1220-1233. [CrossRef]

39. Bibel, D.J.; Crumrine, D.A.; Yee, K.; King, R.D. Development of arthrospores of Trichophyton mentagrophytes. Infect. Immun. 1977, 15, 958-971. [CrossRef]

40. Hashimoto, T.; Blumenthal, H.J. Survival and resistance of Trichophyton mentagrophytes arthrospores. Appl. Environ. Microbiol. 1978, 35, 274-277. [CrossRef] [PubMed]

41. Weigl, E.; Hejtmanek, M. Differentiation of Trichophyton mentagrophytes arthrospores controlled by physical factors. Mycoses 1979, 22, 167-172. [CrossRef] [PubMed]

42. Wright, L.R.; Scott, E.M.; Gorman, S.P. Spore differentiation in a clinical strain of Trichophyton mentagrophytes. Microbios 1984, 39, 87-93.

43. Yazdanparast, S.A.; Barton, R.C. Arthroconidia production in Trichophyton rubrum and a new ex vivo model of onychomycosis. J. Med. Microbiol. 2006, 55, 1577-1581. [CrossRef]

44. Farnoodian, M.; Yazdanparast, S.A.; Sadri, M.F. Effects of environmental factors and selected antifungal agents on arthroconidia production in common species of Trichophyton genus and Epidermophyton floccosum. J. Biol. Sci. 2009, 9, 561-566. [CrossRef]

45. Alshahni, M.M.; Yamada, T.; Yo, A.; Murayama, S.Y.; Kuroda, M.; Hoshino, Y.; Ishikawa, J.; Watanabe, S.; Makimura, K. Insight into the draft whole-genome sequence of the dermatophyte Arthroderma vanbreuseghemii. Sci. Rep. 2018, 8, 15127. [CrossRef]

46. De Vuyst, E.; Charlier, C.; Giltaire, S.; De Glas, V.; Lambert de Rouvroit, C.; Poumay, Y. Reconstruction of normal and pathological human epidermis on polycarbonate filter. Methods Mol. Biol. 2014, 1195, 191-201. [PubMed]

47. Paugam, A.; L'Ollivier, C.; Viguie, C.; Anaya, L.; Mary, C.; de Ponfilly, G.; Ranque, S. Comparison of real-time PCR with conventional methods to detect dermatophytes in samples from patients with suspected dermatophytosis. J. Microbiol. Methods 2013, 95, 218-222. [CrossRef] [PubMed]

48. Minner, F.; Poumay, Y. Candidate housekeeping genes require evaluation before their selection for studies of human epidermal keratinocytes. J. Investig. Dermatol. 2009, 129, 770-773. [CrossRef]

49. Faway, E.; Thiry, M.; Mignon, B.; Poumay, Y. Experimental models of dermatophytosis. In Dermatophytes and Dermatophytosis; Bouchara, J., Nenoff, P., Gupta, A.K., Chaturvedi, V., Eds.; Springer: Cham, Switzerland, 2021; pp. 135-160.

50. Chin, B.; Knight, S.G. Growth of Trichophyton mentagrophytes and Trichophyton rubrum in increased carbon dioxide tensions. J. Gen. Microbiol. 1957, 16, 642-646. [CrossRef]

51. Emyanitoff, R.G.; Hashimoto, T. The effects of temperature, incubation atmosphere, and medium composition on arthrospore formation in the fungus Trichophyton mentagrophytes. Can. J. Microbiol. 1979, 25, 362-366. [CrossRef]

52. Gupta, A.K.; Ahmad, I.; Porretta, M.; Summerbell, R.C. Arthroconidial formation in Trichophyton raubitschekii. Mycoses 2003, 46, 322-328. [CrossRef]

53. Laurent, A.; Monod, M. Production of Trichophyton rubrum microspores in large quantities and its application to evaluate amorolfine/azole compound interactions in vitro. Mycoses 2017, 60, 581-586. [CrossRef] [PubMed]

54. Rippon, J.W.; Scherr, G.H. Induced dimorphism in dermatophytes. Mycologia 1959, 51, 902-914. [CrossRef]

55. Zurita, J.; Hay, R.J. Adherence of dermatophyte microconidia and arthroconidia to human keratinocytes in vitro. J. Investig. Dermatol. 1987, 89, 529-534. [CrossRef] [PubMed] 